\title{
APROXIMACIÓN AL SISTEMAREGIONAL DE INNOVACIÓN VASCO Y SU PAPEL EN LA TRANSFORMACIÓN DEL MODELO PRODUCTIVO
}

\author{
Esther Gil Álvarez \\ Departamento de Geografía. Universidad de Valladolid \\ esther@fyl. uva.es
}

\section{RESUMEN}

Euskadi es una de las regiones que más y mejor ha resistido comparativamente la llegada de la crisis actual, debido en parte al desarrollo de una economía más estable y sólida que otras zonas del país durante décadas anteriores. Siendo una de las últimas comunidades en entrar en recesión, ha sostenido desde hace años una apuesta continua por la innovación, viendo en ella una potente palanca de transformación económica y social. Partiendo de esta premisa, este trabajo analiza el papel que su sistema de innovación ha tenido en este proceso, valorando tanto su composición y trayectoria, como sus principales logros y posibilidades de mejora.

Palabras clave: crisis económica, transformación productiva, sistema de innovación, País Vasco.

\section{ABSTRACT}

Euskadi is one of the regions that more and better comparatively resisted the crisis today, due in part to the development of an economy more stable and robust than other parts of the country during previous decades. Being one of the last communities in entering recession, it has held for years a continuous commitment to innovation, seeing in it a powerful lever for economic and social transformation. Starting from this premise, we analyze the role that its innovation system has played in this process, assessing both their composition and path, as its main achievements and possibilities for improving.

Keywords: economic crisis, productive transformation, innovation system, Basque Country.

Fecha de recepción: septiembre 2014.

Fecha de aceptación: julio 2015. 


\section{INTRODUCCIÓN}

Es indudable que la transformación de la estructura productiva constituye un rasgo consustancial al desarrollo económico de los pueblos, existiendo habitualmente una relación positiva entre crecimiento económico y transición del modelo productivo. Pues a medida que los territorios progresan económicamente y avanzan en su nivel de competitividad, van variando asimismo los factores y agentes clave en los que radica su capacidad, evolucionando las actividades económicas y siendo reemplazadas por otras, con frecuencia más complejas y de mayor valor añadido. Esta exigencia de transformación inherente a todo sistema basado en una economía de mercado, adquiere un cariz más urgente en los momentos de crisis económica, actuando ésta como un acelerador de las transformaciones que debe acometer la economía, partiendo de la máxima de que cuanto antes se efectúen tales, más rápida y segura resultará la salida de la misma.

En el contexto de inquietud territorial actual por la transición del modelo productivo y de competitividad subyacente en los países y regiones, conviene hacer especial mención al caso vasco, pues lo que parecía hace unos años una pasajera interrupción, una ruptura coyuntural limitada a unos pocos sectores (inmobiliario y financiero), se ha revelado claramente como un auténtico cambio de paradigma, una crisis con raíces estructurales que ha trastocado las lógicas de funcionamiento de los mercados, la organización de la producción y demanda globales, todo lo cual se ha saldado con efectos visibles en numerosos ámbitos, especialmente en lo concerniente al desempleo, siendo necesario diseñar una nueva estrategia capaz de liderar la nueva realidad imperante (Orkestra, 2013; Albertos y Sánchez, coords., 2014).

Y es que, en efecto, pese a la especial virulencia con que la recesión iniciada a mediados de 2008 ha azotado prácticamente a toda España en su conjunto, provocando la destrucción de miles de puestos de trabajo y generando cifras de paro escandalosas (con tasas medias de desempleo en torno al 25 y 26\% (EPA, varios años), las investigaciones realizadas evidencian que el País Vasco ha mostrado una capacidad de resistencia superior (V. Apartado I). En la explicación de este proceso y junto a otros factores que más adelante se apuntan, destaca como un elemento diferenciador, su mayor potencial innovador, y relacionado con ello, la importancia concedida a la I+D+i en el contexto del conjunto de medidas impulsadas por los poderes públicos vascos de cara a inducir una transformación productiva enfocada hacia la sociedad y economía del conocimiento; materializada entre otros muchos aspectos (planes, políticas, etc.) en la paulatina gestación y consolidación de uno de los Sistemas Regionales de Innovación (SRI en adelante) más reputados a nivel nacional y mundial (Gil, 2014; Funcas, 2013; Morgan, 2013; Jorda et al, 2014).

Dada su importancia en el texto, y ahondado brevemente en el término (SRI) revisando la literatura existente al respecto, puede afirmarse que, en líneas generales y de forma simplificada, los SRI son estructuras que generan, desarrollan, transfieren y comercializan innovaciones que mejoran la capacidad competitiva de una región, creando con ello riqueza y bienestar en la sociedad. Su origen se remonta a fines de los ochenta y principios de los noventa (Porter, 1990; Lundwall, 1992; Nelson, 1993; Koschatzky, 1997), siendo este modelo el resultado de la integración de varios enfoques teóricos: medio innovador/distrito industrial, polos de crecimiento (Perroux, 1955), teoría de clusters(Porter, 1990; 1999), proceso de aprendizaje colectivo (Koschatzky, 2000) y teoría del crecimiento. Con todo, y 
con el tiempo se ha enriquecido el debate teórico al respecto (V. Doloreux, 2002; Anderson y Karlsson, 2004; Navarro, 2009, etc.). Habitualmente, se conforman en torno a sendos subsistemas de generación y explotación de conocimiento, que interactúan entre sí y se hallan insertos en un marco socioeconómico y cultural con un subsistema de política regional (característico del SRI vasco). Normalmente cuentan con dos elementos clave: de un lado, sistemas de empresas industriales y de servicios avanzados, públicos y privados, que pueden tener un carácter local y son generadores de cambios económicos y tecnológicos debido a su capacidad de absorción del conocimiento externo y a la creación y desarrollo de tecnologías, ciencia e innovaciones en un territorio determinado; Y de otro, instituciones, fundamentales igualmente para desarrollar e incrementar la capacidad innovadora y convertir al territorio en un activo relacional (Boschma, 2005).

Bajo estas premisas, el presente texto posee un propósito y/o hipótesis de partida de carácter doble e interrelacionado: Por un lado, y partiendo del contexto señalado, valorar el papel que dichas infraestructuras y agentes integrantes de la Red Vasca de Ciencia y Tecnología (RVCTI) han desempeñado en la transferencia de conocimiento y en la generación de capacidades en sus empresas para acometer mejor el proceso de transformación productiva apuntado y sortear con éxito los embates de la crisis (actual). Y por otro, tratar de ir un poco más allá y verificar (o no) en la medida de lo posible y a partir de la aportación de evidencias, si verdaderamente, esa función y ese esfuerzo inversor en I+D realizado ha tenido un efecto y unos resultados relevantes en la relativa mejor respuesta al ciclo recesivo que ha experimentado la región en el contexto referenciado.

La elección del tema y objetivos del artículo responden a la premisa de que entre los factores que influyen en el cambio del modelo productivo, para que se pueda dar una transformación es fundamental disponer de recursos humanos cualificados y conocimiento, algo que las empresas, en especial las pymes, claramente dominantes en la economía vasca, por sí solas no son capaces de generar en la medida necesaria. Y es que las infraestructuras de conocimiento del territorio, al proveer de capital humano capacitado y prestar servicios de I+D e innovación a las empresas, complementan las capacidades desarrolladas por éstas y pueden desempeñar un papel clave en el proceso de transformación económica. A este respecto, cada vez es más patente que, en la explicación de las diversas trayectorias recientes y la desigual capacidad para generar ventajas competitivas en los territorios, uno de los argumentos que ha ganado en importancia es el que relaciona esa mejor evolución con su posicionamiento en la sociedad y economía del conocimiento (Méndez et al, 2011; Méndez, 2013). Con todo, conviene apuntar que el territorio no es algo homogéneo e incluso en una región con un alto grado de cohesión como es la vasca se observan realidades claramente diferentes a nivel provincial, comarcal e incluso municipal. De ahí que, sobre la base del diagnóstico de su Red de Ciencia y Tecnología, centramos también nuestra atención en la perspectiva geográfica, analizando la dimensión espacial de este proceso. Para ello, y tras un primer apartado en el que se valora la competitividad diferencial de la región vasca en el contexto nacional (e incluso europeo), insistiendo brevemente en las condiciones de partida y políticas implementadas, se exponen a continuación los rasgos fundamentales y dinámica evolutiva de su SRI en tanto pilar básico en el elenco de las medidas impulsadas desde la Administración Vasca en aras a modernizar y dotar de competitividad a su tejido productivo. Con este fin, se hace asimismo un breve repaso a los principales planes de ciencia y tecnología y actuaciones en la materia 
que dan soporte a la innovación en la región, profundizando paralelamente en la caracterización de los componentes del sistema y su distribución espacial. Finalmente, en un tercer apartado, y sobre la base de la utilización de las estadísticas e informes existentes, se tratan de apuntar, con una visión crítica y comparada, los principales logros y evidencias obtenidos desde diferentes perspectivas de análisis en relación con el propósito del trabajo, así como los cambios que habría que introducir para optimizar más y mejor su papel de proveedores de capacitaciones y conocimientos, no sólo tecnológicos, sino también organizativos y de otro tipo, que las empresas precisan captar del exterior para poder innovar y transformarse.

El texto toma como base fundamental la revisión de la literatura existente sobre el tema (artículos, monografías, actas, etc.), así como la información y explotación de los datos contenidos en diversos informes y estadísticas fundamentalmente del INE (INEbase), EUSTAT (Instituto Vasco de Estadística), Eurostat, OCDE, Instituto Vasco de la Competitividad, etc.

\section{LA COMPETITIVIDAD DIFERENCIAL DEL PAÍS VASCO EN EL CONTEXTO NACIONAL Y EUROPEO}

Como es sabido en el segundo trimestre de 2008 alcanza también a España la gran recesión. Así, y en apenas cinco años el PIB descendería en España un 7\%, la destrucción de empleo alcanzaría al $18 \%$ de los ocupados, y la tasa de paro se situaría por encima del $26 \%$ de la población activa (Funcas, 2013). Sin embargo, la entrada en recesión económica no se ha producido a la vez en todas las regiones del Estado y la intensidad con la que se ha padecido su efecto no ha sido homogénea. Diversos estudios sobre el alcance de la crisis en España desde un enfoque territorial han puesto de manifiesto que, dentro de un período común regresivo que afecta a todas las regiones, existen notables diferencias entre ellas. El análisis de su conducta y actuaciones durante la crisis desvela, junto a importantes problemas estructurales en algunas, condiciones de partida así como políticas y estrategias diferenciales en la explicación de los contrastes en su desempeño económico. A este respecto, y en un ejercicio sintético, el análisis de los datos del INE (Contabilidad Nacional y Regional) relativos al empleo, afiliación a la Seguridad Social, paro y producción industrial entre 2008 y 2013 evidencian el impacto de la recesión en España y las diferencias entre sus territorios (Bandrés y Gadea, 2013; Albertos y Sánchez, coords., 2014). La combinación de todos esos indicadores sitúan a la Comunidad Valenciana, Castilla-La Mancha y Andalucía como el grupo más castigado por la crisis, con Murcia y Canarias en una posición cercana aunque no muy alejada de aquellas con un impacto intermedio que se corresponden con Aragón, Cataluña, La Rioja y Baleares. Con todo, el grupo con menor impacto está formado por el País Vasco, Navarra y Madrid siendo la vasca la comunidad menos afectada de todas; con Galicia y Castilla y León también incluidas si bien cercanas al grupo intermedio.

Las regiones con menor incidencia de la crisis cuentan en líneas generales con un denominador común vinculado a su mayor peso industrial, mejor dotación de capital humano, menor tasa de paro antes de la recesión y una apertura exterior superior; Además, por regla general, la trayectoria de su crecimiento suele ser también bastante homogénea en el tiempo. Todo lo contrario sucede con las regiones que experimentan un mayor impacto de la recesión; Una recesión que apenas ha modificado la ordenación de las regiones atendiendo a su indicador económico más representativo, la renta per cápita, de manera que, según los 
últimos datos disponibles, el País Vasco continúa encabezando la clasificación, seguida de cerca por Madrid, Navarra, Cataluña, Aragón y La Rioja, que se mantienen en las primeras posiciones (Inebase, 2014).

Este impacto diferencial de las regiones españolas frente a la crisis, y las desiguales consecuencias territoriales asociadas a la misma, incita a profundizar sintéticamente en el exitoso caso vasco, pues en efecto, y como revela la literatura existente, su modelo de crecimiento presenta rasgos diferentes de la media nacional y, en especial, de las regiones más castigadas, además de constituir, como se apuntó en la introducción, el espacio menos afectado comparativamente. Así, y como se recoge en numerosos estudios, el crecimiento del PIB per cápita en España durante la fase de expansión económica se sustentó principalmente en el aumento de la tasa de empleo, con un registro muy mediocre del crecimiento de la productividad. En cambio, en el País Vasco el aumento de la tasa de empleo estuvo acompañado por un crecimiento de la productividad muy superior a la media nacional. De igual modo, con la crisis, el fuerte ajuste del empleo ha elevado la productividad aparente del trabajo en el conjunto de España, mientras en el País Vasco el empleo ha caído menos y la productividad también ha crecido a tasas más bajas (Aranguren et al., 2013).

Los resultados del análisis regional comparativo que estos autores realizan a escala no sólo nacional sino también europea -tomando como base una serie de indicadores económicos y de innovación-, reflejan que Euskadi se caracteriza sobre todo por unas determinadas condiciones de partida entre las que destacan, en comparación con el promedio nacional, un PIB per cápita más elevado (de hecho, el más elevado de España), un empleo más cualificado (terciario), un sector industrial muy potente y en especial, una mayor capacidad de innovación (sobre este aspecto se hablará en detalle más adelante). Además, el País Vasco ha mostrado una menor dependencia del sector de la construcción en la composición de su estructura productiva, siendo éste otro elemento diferenciador, lo cual permite entender, junto a otros agentes (peculiar sistema de gobernanza, sistema fiscal distinto, etc.) su mejor comportamiento relativo frente a la crisis (Gil, 2014; Jorda et al, 2014, Gil, 2014) ${ }^{1}$. Todo ello

1 Abundando en esta idea, en el libro Geografía de la crisis económica en España (2014), coordinado por Albertos y Sánchez, Gil ofrece en el capítulo titulado «El País Vasco y su capacidad diferencial de resistencia a la crisis» una radiografía sintética de la economía vasca durante la recesión económica entre 2008 y 2012 seleccionado indicadores expresivos relacionados con la evolución de su competitividad en el periodo señalado que avalan su mejor desempeño económico relativo. Así lo atestigua su tasa de paro que, pese a la fuerte subida del último año evaluado (2012), se situaba en el 14,88\% frente al 25,02\% de media estatal (EPA, 2012); su nivel de deuda pública (10,7\% del PIB), muy inferior a la media de las comunidades autónomas (15,9\%, Banco de España, 2012); O su PIB per cápita $(31.058 €$ frente a $23.054 €$ del promedio nacional, el más alto de todo el Estado (INE, 2012). Por otro lado, la evolución y composición de su estructura sectorial, también refleja un protagonismo superior de la industria respecto a la media del conjunto del país (aportación al VAB del 24, 5\% en la CAPV frente al 15,6\% de media nacional -año 2011-) y una dependencia menor de la construcción (7,8\% frente al 10,1\% respectivamente) así como una estructura del mercado laboral con casi un $23 \%$ de su población ocupada en la industria frente al 13,5\% en el sector del «ladrillo». Esto es destacable porque el sector industrial suele presentar una productividad o generación de VAB superior, como media, a la de los otros grandes sectores, explicando, en parte, el buen comportamiento que en la productividad del total de la economía y en el PIB per capita muestra el País Vasco, con valores superiores a España e incluso a la media de la UE-27. En cuanto al grado de internacionalización, desde el 2000 hasta el 2011 el volumen de las exportaciones vascas ha sido siempre superior al promedio español con diferencias que oscilan entre los ocho y los diez puntos de media, contribuyendo con ello a un mayor sostenimiento de su generación de riqueza. Destacan las anualidades correspondientes a 2008 y 2011 como los períodos en los que, pese a la intensidad de la crisis a nivel nacional y europeo, Euskadi logra máximos con un 29,9\% y un $31,5 \%$ de ventas sobre su PIB. Algo 
obliga a profundizar así mismo en las estrategias y políticas públicas desarrolladas, en buena medida claves en la génesis de las ventajas competitivas de esta región. Pues, en efecto y como ya se ha avanzado, frente a las políticas de devaluación interna basadas en la reducción de los costes laborales unitarios, dominantes en el conjunto del país, Euskadi ha mantenido el pulso inversor en I+D (situándose en la primera posición de las regiones españolas), con especial protagonismo por parte del sector empresarial privado frente al menor peso relativo del gasto público. También cabe destacar la apuesta por la industria como base para el impulso de la competitividad de su economía, con notables progresos en la diversificación sectorial y en la formación de clusters, buscando así la especialización en manufacturas de nivel tecnológico medio-alto; y además la continuidad en el tiempo de las políticas horizontales de formación de activos específicos, como la innovación, el capital humano, las infraestructuras físicas y los activos institucionales (Aranguren et al, 2013) ${ }^{2}$.

\section{EL SISTEMA DE INNOVACIÓN VASCO Y SU FUNCIÓN EN LA TRANSFORMACIÓN PRODUC- TIVA DE LA REGIÓN}

Como se acaba de señalar, la Comunidad Autónoma del País Vasco (CAPV) es una de las regiones que más y mejor ha resistido comparativamente la llegada de la crisis actual. En la explicación de este proceso, obviamente coexisten un buen cúmulo de factores, pero junto a la aplicación de diversas políticas y medidas, y a la existencia de determinadas condiciones de partida, no cabe duda de que el apoyo sostenido y continuado a la innovación durante más de tres décadas por parte de empresas e instituciones ha sido clave, además de un elemento diferenciador en el conjunto, viendo en la I+D una potente estrategia de transformación económica y social capaz de posicionar a la CAPV entre las regiones españolas y europeas más avanzadas.

Cierto es que el País Vasco no partía de cero en esta andadura, habiendo experimentado ya otra fuerte crisis. En los años setenta y ochenta, la reestructuración masiva de la economía en sectores como el acero, la construcción y la máquina-herramienta tuvieron como consecuencia importantes tasas de desempleo y emigración. La región se convirtió así mismo en un modelo por el éxito de su llamada «Primera Gran Transformación», un proceso destinado a reestructurar su industria y aumentar su competitividad a través de unas políticas industriales específicamente adaptadas, entre las que estaba ya la creación del sistema de centros

que no sucede en el conjunto de la economía española que, con alguna que otra excepción, ve descender el porcentaje de sus ventas desde el 2000 logrando tan solo en 2011 recuperar los niveles iniciales, lo que explica y justifica al propio tiempo la mayor competitividad de la economía vasca en el exterior. Así, en 2011 en el ámbito vasco el $31,5 \%$ de su economía se exportaba frente al 20\% de España en 2011 (Gil, 2014, pp. 497-539).

2 Con todo, resulta innegable esgrimir que la mejor capacidad diferencial vasca se ha ido aminorando en los últimos tiempos, al socaire de la contracción de la demanda interna, de los recortes monetarios auspiciados por el sector público o como consecuencia de insuficiencias en la gestión empresarial, provocando la quiebra de numerosas firmas o su entrada en concurso de acreedores y posterior liquidación (p. e Fagor Electromésticos puntal del Grupo cooperativo Mondragón, recientes casos de Candy Electrodomésticos (Bergara), Muebles Azcue (Azpeitia), etc.), acortándose la brecha que separaba ambos conjuntos espaciales y siendo necesario por tanto, acometer nuevas estrategias o redefinir líneas de actuación preexistentes de cara a la recuperación de los estadios de competitividad previos. Por otro lado, es innegable que, en el elenco de actuaciones implementadas por la Administración Pública Vasca, se han cometido también errores (V. fallidos proyectos relacionados con Hiriko o el tema de las ayudas a Epsilon Euskadi). 
tecnológicos (CT’s), puntal básico del sistema de innovación como se verá. Pero el triunfo del modelo anterior no garantizaba las condiciones necesarias para el éxito futuro, pues la transformación se asentaba principalmente sobre formas de innovación incrementales y de reducción de costes por parte de las empresas locales (OECD, 2011). En este contexto, y además de apoyar estas innovaciones, el País Vasco se plantea la mejora de las condiciones necesarias para que el conocimiento (incluida la ciencia) actúe como motor de la innovación; intentando pasar de un modelo de innovación incremental y de reducción de costes a un modelo más intensivo en conocimiento. También forma parte de esta tendencia más novedosa la creación de oportunidades para que la innovación contribuya al cumplimiento de objetivos y necesidades sociales. Conscientes de esta realidad y bajo el paraguas de diferentes planes, especialmente de los recientes planes de Ciencia y Tecnología (CTI 2010), continuado por el plan CTI del 2015, el País Vasco está intentando sentar las bases de una «Segunda Gran Transformación Económica Regional» apostando por un nuevo modelo productivo que descanse en la economía de la innovación y del conocimiento (OECD, 2011; Gobierno Vasco, 2011).

De ahí por tanto, la pertinencia de profundizar en este aspecto concreto, pues la CAPV se ha singularizado tradicionalmente por ir construyendo y gestionando de forma continuada una sólida infraestructura de conocimiento científico y tecnológico que le ha otorgado una ventaja distintiva clara con respecto a otros territorios. Así, ha implementado su propia política de ciencia, tecnología e innovación (CTI), una de cuyas señas de identidad más notorias reside en la generación y consolidación de su SRI que, junto con el de Cataluña, CA de Madrid o Navarra, está considerado entre los mejores del estado y goza de un gran reconocimiento (OECD, 2011; Jorda et al., 2014) ${ }^{3}$.

\section{III.1. Caracterización del sistema de innovación (SRI) vasco}

Tal como se acaba de apuntar, en los últimos 30 años el País Vasco ha desarrollado una importante red de ciencia y tecnología, contando con un conjunto de organizaciones y agentes institucionales y empresariales que conforman el llamado SRI vasco. A este respecto, y a partir de los resultados de un análisis cluster sobre los distintos sistemas regionales de innovación en España entre 2007 y 2011, el SRI vasco, junto con el navarro se encuadran dentro de los llamados sistemas de excelencia basados en el subsistema empresarial, posicionándose como los más avanzados a nivel de todo el país en la actualidad (Jordá et al, 2014: 279). Pues en efecto, según los últimos datos disponibles, ambos reúnen el 11,27\% de las empresas innovadoras del total nacional, el 13,49\% de los gastos de innovación empresarial y el $12,60 \%$ de la inversión en I+D. El principal motor de estos sistemas es el sector empresarial, que supone el $18 \%$ de los gastos internos totales realizados en España, mientras que sólo recibe el 6,10\% de la Enseñanza Superior y el 4,40\% de la Administración Pública. Los resultados científico-tecnológicos y económicos de sus sistemas se observan en la alta capacidad para patentar vía internacional $(12,02 \%)$ y nacional $(10,16 \%)$, para generar valor añadido en manufacturas de alta y media tecnología (17,80\% del VAB total de estos sectores

3 Con todo, justo es decir que esta compleja y sofisticada red de innovación adolece de determinadas debilidades y problemas que, así mismo merece la pena ser comentados en estas páginas. 
en España) y de internacionalización sobre todo en manufacturas de media-alta tecnología (17,67\% de la exportación total de estos sectores). Se sustentan en el tejido empresarial y en el conocimiento acumulado por los diferentes agentes, así como en las características del territorio y sus capacidades específicas (ibid).

En una aproximación concreta al SRI vasco, éste se ha distinguido tradicionalmente de las regiones españolas y de la OCDE por varias peculiaridades que le otorgan caracteres distintivos frente a otro tipo de organizaciones similares: por un lado, se ha sustentado principalmente en los centros tecnológicos, y en menor medida en el sistema universitario, siendo esta característica un rasgo idiosincrásico frente a otras regiones líderes como Cataluña y Madrid, donde el SRI está basado en el potencial científico de grandes universidades. Se ha señalado ya que la crisis de los setenta hizo más visible la debilidad de los sectores tradicionales de la industria vasca. Una parte importante de los líderes empresariales, organismos públicos y demás grupos locales interesados detectaron la necesidad de reestructurar el sistema productivo e impulsar la competitividad mediante la innovación, el conocimiento y el desarrollo tecnológico. Así pues, los Centros Tecnológicos se crearon en los años ochenta para paliar la brecha tecnológica y mejorar las capacidades tecnológicas de sus sectores industriales. El Gobierno Vasco los apoyó activamente con el objetivo de construir nuevas capacidades a partir de una política clara de oferta tecnológica. El principal instrumento fue el apoyo prestado a los denominados «centros tutelados» mediante la concesión de financiación con base en los objetivos y planes operativos presentados a las autoridades vascas (López-Rodríguez et al., 2010). Con todo, la piedra angular del SRI es la Red Vasca de Ciencia, Tecnología e Innovación-actual RVCTI-, creada en 1997 por el Gobierno Vasco con el objetivo de reunir los esfuerzos de los organismos públicos y privados encargados de impulsar el conocimiento científico, la tecnología y la innovación como medio para mejorar la competitividad de las empresas y contribuir al desarrollo económico y social de la región. Su propósito es desarrollar una infraestructura tecnológica apta para trabajar en red de forma coordinada, orientada al mercado y al cliente y capaz de ofrecer una oferta tecnológica integral al tejido empresarial vasco (Innobasque, 2014). Los miembros de la Red se agrupan según su naturaleza y actividades en diferentes categorías: los citados centros tecnológicos, unidades de I+D empresariales, centros internacionales de transferencia de tecnología y desarrollo, universidades, organismos certificadores y laboratorios de homologación, centros públicos de investigación, parques tecnológicos, centros de empresa e innovación, centros de investigación cooperativa (CIC's), centros de investigación básica y de excelencia (BERC's), etc. Estos dos últimos son de carácter científico y se han creado en la última década (desde 2005) para mejorar el tejido científico e investigador del SRI vasco.

La RVCTI se articula en tres grandes subsistemas: a) el científico y universitario (incluyendo universidades y centros de investigación de excelencia (BERC's, CIC's); b) el de innovación y desarrollo tecnológico (centros tecnológicos, centros sectoriales, entidades de certificación y laboratorio, unidades de I+D de empresas, unidades de I+D sanitarias, etc.); y c) el de apoyo a la innovación (parques tecnológicos, intermediarios, etc. (Innobasque, 2014).

En el ámbito científico, Euskadi cuenta en la actualidad con un subsistema de ciencia que ha crecido en capacidades de una manera importante en los últimos años, al ponerse en marcha las nuevas organizaciones apuntadas (los BERC's y los CIC's), y en el que destacan algunos grupos de investigación de primer nivel internacional que han venido a paliar la 
apuntada debilidad de la investigación pública. Con su núcleo principal en la Universidad del País Vasco (UPV), presenta sus mayores capacidades en varias disciplinas asociadas a las Ciencias Experimentales (Física, Química, Ciencias de Materiales y Medicina), apreciándose últimamente una diversificación hacia áreas científicas de carácter transversal e interdisciplinar (Biomedicina, TIC's) y una mayor presencia de las Ciencias Sociales y Humanidades. Cuenta además con un buen conjunto de escuelas de ingeniería y una nutrida oferta de escuelas de negocio. Sus mayores retos pasan por incrementar su productividad científica, proyección internacional y conectividad con el resto de agentes tecnológicos y empresariales de la región (Gobierno Vasco, 2011). Las universidades junto a los organismos de investigación dependientes de la Administración Pública (OPI's) forman el núcleo básico del sistema de investigación científica y desarrollo tecnológico del SRI vasco.

En el ámbito tecnológico, Euskadi cuenta con una potente red de Centros Tecnológicos. Este hecho se pone de manifiesto al considerar que los centros vascos asociados a FEDIT (Federación Española de Entidades de Innovación y Tecnología) suponen más de una cuarta parte de los CT's españoles (Gálvez y González, 2012). En los últimos años los centros tecnológicos han experimentado un proceso importante de consolidación y agrupamiento con la constitución de la Corporación Tecnalia y de la Alianza IK-4. Las dos grandes corporaciones tecnológicas vascas se sitúan entre las mayores de Europa y son representativas, en general, de los retos y las capacidades del tejido empresarial vasco.

Finalmente, y en lo relativo al subsistema de apoyo a la innovación se encuentran los Clusters empresariales, los Parques Tecnológicos (PT) (la red de parques tecnológicos del País Vasco es pionera en España) y los Centros de empresas e innovación (CEI's) en los que se ubican las empresas vascas más intensivas en I+D+i. Constituyen en la actualidad verdaderos nodos de encuentro entre el conocimiento científico y empresarial y se configuran como entornos óptimos para la innovación. El PT de Vizcaya, en Zamudio, el de Álava en Miñano o el de San Sebastián en el área de Miramón -con el proyecto de ampliación en el área Galarreta de Hernani (Proyecto ORONA), junto con el Polo de innovación Garaia (Arrasate), o los centros de empresas en innovación como BIC BERRILAN, etc., constituyen muestras expresivas de la intensa apuesta por dotar al territorio de las infraestructuras necesarias para la localización y el desarrollo de agentes e instituciones vinculados con el proceso innovador ${ }^{4}$. Todo este entramado de agentes y organizaciones es el resultado de diferentes acciones implementadas a través de diversos planes y políticas en materia de competitividad industrial y especialmente, en el ámbito de la ciencia y la tecnología impulsados en especial por el Gobierno Vasco, a través de sus Departamentos de Industria y Educación.

4 Asimismo y para estimular el trabajo en red y la gobernanza el SRI vasco se está dotando progresivamente de otras organizaciones claves en la gestión como el Consejo Vasco de Ciencia, Tecnología e Innovación en tanto que órgano superior de participación, asesoramiento y liderazgo de la política científica, tecnológica, de investigación y de innovación; el Consejo Vasco de Investigación como entidad de observación, coordinación, promoción y asesoramiento de la investigación; el Comité de Ciencia y Tecnología como órgano interdepartamental dentro del Gobierno Vasco que actúa como coordinador de la definición, diseño y puesta en marcha de las políticas científica y tecnológica. Y de forma relevante, con otras agencias de innovación y atracción de talento (Ikerbasque) como elementos de coordinación y soporte (Innobasque). 


\section{III.2. La política de Ciencia y Tecnología (CTI) en el País Vasco: Fases principales}

La política de Ciencia, Tecnología e Innovación tiene un recorrido en Euskadi que se remonta a más de tres décadas desde su inicio a principios de los 80 , habiéndose caracterizado por su continuidad a lo largo del tiempo en cuanto a sus grandes objetivos y a muchas de sus actuaciones. El denominador común de esta política reside en unas bases estratégicas adaptadas en cada momento a la situación económica del entorno. Su desarrollo se ha realizado desde una visión global que, de un lado, trataba de responder a las necesidades competitivas industriales a corto plazo de la economía vasca, al tiempo que abría áreas de diversificación industrial desde las capacidades propias; y de otro, ha desarrollado estrategias a medio-largo plazo y pretendido construir en el tiempo un SRI articulado y cada vez más abierto al mundo (Gobierno Vasco, 2011: 40). Estas medidas han evolucionado desde una concepción inicial basada sobre todo en la tecnología, la integración posterior de la ciencia y la consideración final de la innovación como clave para transformar en resultados el conocimiento generado. De esta manera se ha pasado de un modelo lineal de innovación a uno no-lineal, de unas políticas de oferta a unas políticas combinadas de oferta y demanda, para promover finalmente políticas orientadas a resultados. Las distintas fases y elementos más importantes de la política de ciencia, tecnología e innovación desarrollada en el País Vasco desde los años ochenta se muestran en la figura 1. Cada etapa corresponde a un período en el que los principales instrumentos de la política de innovación se centran en aquellas acciones factibles que, a su vez, permiten eliminar los principales obstáculos que limitan las posibilidades de aplicar con cierta garantía de éxito las medidas características de una etapa más avanzada. Como se expone en la figura, se pueden distinguir tres grandes etapas lógicas y cronológicas en la evolución de la política de ciencia y tecnología del País Vasco: 1) El período 1980-1996 puede considerarse como un período de creación de capacidades -«política de oferta»- en el que se construyen los cimientos de la apuntada RVCTI, orientando todas las medidas a sentar las bases, fundamentos e infraestructuras de la nueva economía que surge de la reestructuración económica y social del País Vasco y que tiene su máximo exponente con el apoyo y tutela de los centros tecnológicos; 2) El período 1997-2005 es un período de «política combinada de oferta y demanda» en el que los esfuerzos se dirigieron fundamentalmente a la consolidación de la RVCTI (antigua SARETEK), creada en 1997, y a la atención de las prioridades en conocimientos tecnológicos e innovación de los principales actores empresariales y sociales. 3) Finalmente, el período 2006-2015, en el contexto de la Estrategia Vasca 2015, y teniendo como base los planes de ciencia, tecnología e innovación de 2010 y 2015, podría denominarse como «política orientada a resultados», cuyos objetivos principales están dirigidos tanto a la diversificación del tejido empresarial como a la consecución de resultados tangibles en términos de ciencia, tecnología e innovación de cara a afrontar los retos de la creciente globalización y de la competencia de nuevos países miembros de la UE (López Rodríguez et al., 2010).

El Plan de Ciencia, Tecnología e Innovación 2010 (PCTI2010) apuesta por situar a Euskadi como un referente en innovación en Europa. Convive en el tiempo con el Plan de Competitividad e Innovación Social 2006-2009. De igual forma, en 2007 se pone en marcha el Plan Universitario 2007-2010, primer plan integral del Sistema Universitario Vasco, que apuesta por su inserción en el Espacio Europeo de Educación Superior y en el Espacio Euro- 


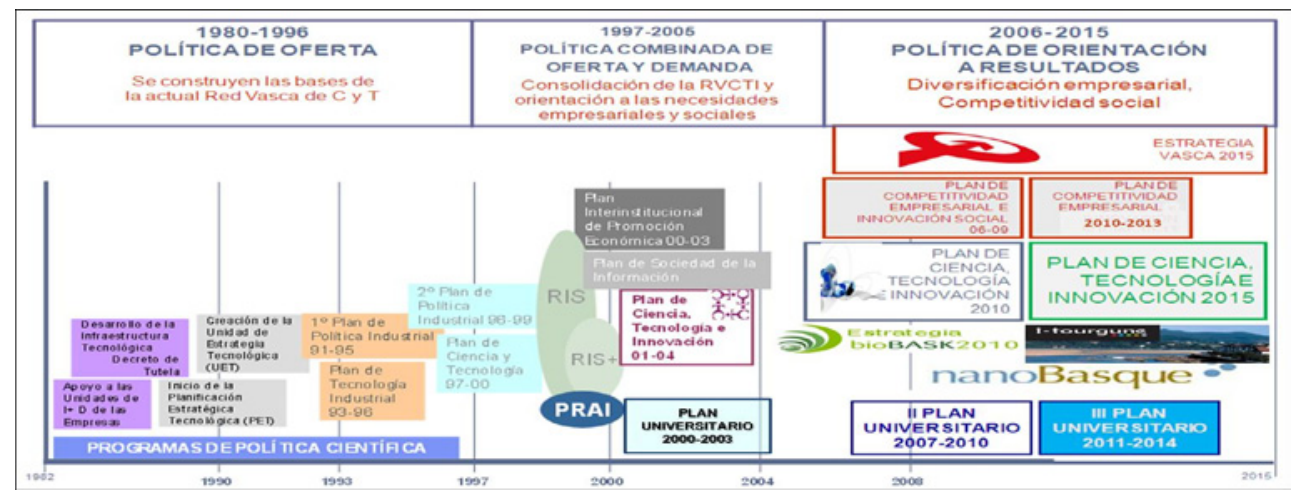

Fuente Gobierno Vasco: Plan Ciencia y Tecnología e Innovación 2015, 2011: 41.

peo de Investigación. El denominador común de este período es una mayor orientación de la política de ciencia y tecnología a la obtención de resultados. Por su parte y con estas bases, el PCTi 2015 reconoce nuevamente la creciente importancia e impacto de la ciencia y la tecnología en una sociedad que camina hacia la economía del conocimiento, planificando un futuro ambicioso, en casi una decena de objetivos, que van a exigir un gran esfuerzo a todos los agentes del sistema de ciencia y tecnología (Gobierno Vasco, 2011). El plan de 2015 busca consolidar un Sistema de Ciencia y Tecnología de segunda generación que tiene ahora el reto de aumentar su productividad científica y tecnológica y su contribución a la creación de valor en forma de nuevos productos y servicios y de nuevas empresas, y de ganar presencia y protagonismo a nivel internacional, acompañando a las empresas vascas.

\section{PRINCIPALES LOGROS DE LA POLÍTICA DE INNOVACIÓN EN EL PAÍS VASCO}

Como se ha comentado, uno de los principales retos del País Vasco ha sido y es convertirse en una economía competitiva basada en el conocimiento y la innovación. Está ampliamente aceptado que el desarrollo de una zona o país depende en gran proporción de sus avances en materia científica y tecnológica. Por tanto, la inversión destinada a I+D+i es uno de los factores clave o Inputs que inciden en la competitividad territorial y constituye uno de los pilares fundamentales para conseguir un crecimiento económico sostenible (si bien no el único). En este apartado se examinan, no sólo parámetros como la inversión sino también los principales resultados de las actividades de investigación e innovadoras desarrolladas por los principales agentes u organizaciones que componen el sistema de innovación de Euskadi en los últimos años. Con estos fines, se realiza en primer lugar una panorámica de conjunto sobre las magnitudes e indicadores básicos que presenta la investigación y el desarrollo tecnológico en el País Vasco a partir de los últimos datos disponibles, para obtener una visión, en la medida de lo posible comparada, a nivel nacional y europeo de las mismas. A continuación, nos centramos en los resultados que presenta la región en su conjunto descendiendo a escala provincial 
y comarcal con el fin de detectar efectos diferenciales al respecto. Finalmente, focalizamos nuestra atención en los principales agentes del sistema, comenzando por las propias empresas, para seguidamente valorar los datos relacionados con las principales infraestructuras del conocimiento existentes. El análisis se basa fundamentalmente en la explotación de las estadísticas de actividades de I+D e innovación del Ine (Inebase), Eustat, y de Eurostat. Estas estadísticas ofrecen datos de actividad innovadora y son las que proporcionan información más completa sobre los principales agentes del sistema de innovación. Del mismo modo, y con carácter complemetario y/o de apoyo, nos hemos servido de numerosos informes de la OCDE, el Instituto Vasco de Competitividad, COTEC, etc. para realizar una valoración al respecto.

\section{IV.1. Visión general comparada de los resultados de la I+D en el País Vasco}

Una visión general y comparada sobre los resultados de la I+D en el País Vasco muestra que, en lo que se refiere al gasto dedicado a actividades de investigación y desarrollo y esfuerzo inversor (gasto en relación al PIB), el posicionamiento de Euskadi es muy positivo, presentando un nivel relativo muy superior al promedio español, e incluso como más adelante se mostrará, en la línea mostrada por la media exhibida en la UE-27. En efecto, tal y como queda reflejado en la Tabla 1 el País Vasco ha realizado en 2012 un gasto en I+D+i de 1.431 millones de $€$ (1. 372 millones según Eustat), lo que representa el 2,19\% de su PIB (2,12\% según Eustat), superando ampliamente la media española, que es del 1,30\% (13.391 millones de $€$ ) e incluso la media del gasto de la UE-27 (2,08\%), Eurostat y Eustat, 2014) $)^{5}$. La perspectiva temporal evidencia el crecimiento anual sostenido de la inversión en I+D en Euskadi durante toda la década desde 2002, incluso durante el periodo comprendido entre 2008 y 2012, confirmando el mejor comportamiento relativo del País Vasco durante la crisis como se indicaba en el capítulo primero. Así, entre 2002 y 2012, el gasto en I+D es realmente alto, en sintonía con la importancia otorgada desde el Gobierno Vasco a la Política de Ciencia y Tecnología con un aumento global del $140 \%$ en periodo apuntado. Esta tendencia de crecimiento se mantiene hasta la actualidad, con tasas de variación positivas todos los años, destacando 2003 (+ del 14\%), 2007 (aumento de casi el 27\%) y especialmente 2011, es decir, en pleno periodo recesivo, con un incremento anual del $7,01 \%$ si bien en un contexto de crecimiento periódico inferior por efecto de la recesión y los recortes presupuestarios asociados. Con todo, y excepto 2010, con un descenso de tres puntos, la inversión ha sido positiva todos los años situándose en 2012 la tasa de variación anual en un $2,43 \%$. El gasto en actividades de investigación y desarrollo tecnológico medido en porcentaje sobre el PIB pasa del 1,29\% del 2002 al 2,19\% de 2012, no dejando de crecer ni un solo momento, habiendo experimentado un aumento global del casi el 70\%. Entre 2008 y 2012 pasa de un 1,98\% a un 2,19\% teniendo todos los años saldo positivo. En el promedio español, partiendo de niveles de inversión relativos muy elevados, incluso más que los mostrados por la CAPV, con una tasa de incremento anual en 2002 del 15,50\% y un gasto de más de 7 millones de $€$, se acusa sobre manera el impacto de la recesión, con tasas de variación anuales negativas casi todos los años a partir de 2008, excepto 2010 que se mantiene prácticamente igual que el

5 Apuntamos aquí también los datos proporcionados por Eustat porque se utilizarán para el análisis de los resultados de la CAPV a nivel intrarregional (Territorios Históricos y comarcas) al ser la mejor e incluso única fuente para la valoración de la I+D a nivel intrarregional (escala comarcal, etc.). Con todo, y para el análisis comparado a escala nacional y autonómico se ha preferido utilizar la misma fuente procedente del INEbase. 
año anterior $(+0,05 \%)$ y donde destaca el desplome en $2011(-2,77 \%)$ y sobre todo en 2012 con una disminución del gasto del casi el 6\%. Además, y como refleja la Tabla 1, que recoge la evolución durante toda la década del esfuerzo inversor en I+D (porcentaje del gasto en I+D en relación con el PIB), la inversión total en I+D en 2012 y su relación con el PIB $(1,30 \%)$ se han retrotraído a los niveles de 2007, es decir, a los existentes antes a los años del inicio de la crisis, evidenciando la mella que, sobre las actividades de I+D+i sufre España por la fuerte disminución inversora en este ámbito.

Tabla 1

EVOLUCIÓN DEL GASTO TOTAL EN I+D, RELACIÓN CON EL PIB Y TASA DE VARIACIÓN ANUAL (2002-2012). ESPAÑA Y CAPV

\begin{tabular}{|c|c|c|c|c|c|c|}
\hline & \multicolumn{3}{|c|}{ ESPAÑ } & \multicolumn{3}{c|}{ CAPV } \\
\cline { 2 - 7 } & Importe & \% PIB & $\begin{array}{c}\text { \% var. } \\
\text { anual }\end{array}$ & Importe & \%PIB & $\begin{array}{c}\text { \% var. } \\
\text { anual }\end{array}$ \\
\hline $\mathbf{2 0 0 2}$ & 7.193 .537 & 0,99 & 15,5 & 581.744 & 1,29 & 3,7 \\
\hline $\mathbf{2 0 0 3}$ & 8.213 .036 & 1,05 & 14,2 & 667.281 & 1,39 & 14,7 \\
\hline $\mathbf{2 0 0 4}$ & 8.945 .761 & 1,06 & 8,9 & 778.443 & 1,51 & 16,7 \\
\hline $\mathbf{2 0 0 5}$ & 10.196 .871 & 1,12 & 14,0 & 829.025 & 1,48 & 6,5 \\
\hline $\mathbf{2 0 0 6}$ & 11.815 .218 & 1,2 & 15,9 & 959.393 & 1,58 & 15,7 \\
\hline $\mathbf{2 0 0 7}$ & 13.342 .371 & 1,27 & 12,9 & 1.216 .726 & 1,87 & 26,8 \\
\hline $\mathbf{2 0 0 8}$ & 14.701 .393 & 1,35 & 10,2 & 1.345 .572 & 1,98 & 10,6 \\
\hline $\mathbf{2 0 0 9}$ & 14.581 .676 & 1,39 & $-0,8$ & 1.346 .984 & 2,06 & 0,1 \\
\hline $\mathbf{2 0 1 0}$ & 14.588 .455 & 1,39 & 0,0 & 1.305 .630 & 2,13 & $-3,1$ \\
\hline $\mathbf{2 0 1 1}$ & 14.184 .295 & 1,33 & $-2,8$ & 1.397 .209 & 2,11 & 7,0 \\
\hline $\mathbf{2 0 1 2}$ & $\mathbf{1 3 . 3 9 1 . 6 0 7}$ & $\mathbf{1 , 3 0}$ & $\mathbf{- 5 , 6}$ & $\mathbf{1 . 4 3 1 . 1 0 8}$ & $\mathbf{2 , 1 9}$ & $\mathbf{2 , 4}$ \\
\hline
\end{tabular}

*a partir de 2002 incluye innovación continua y ocasional; Importes en miles de $€$

Fuente: INE. Estadística sobre actividades de $I+D$, varios años y elaboración propia.

Con todo, esta media nacional oculta situaciones desiguales entre las diversas comunidades autónomas a la luz de las ideas anticipadas en el primer apartado de este texto (Tabla 2). Así, los últimos datos disponibles, sitúan al País Vasco en primera posición en gasto en I+D sobre el PIB $(2,19 \%)$. Junto con Navarra, Madrid y Cataluña son las cuatro regiones situadas por encima de la media nacional (1,30\%). El resto de comunidades están por debajo destacando el escaso peso que en las ciudades autónomas de Ceuta y Melilla y en los archipiélagos insulares posee esta variable. Por otro lado, la estructura del gasto en I+D por parte de los principales agentes del sistema (empresas, Administración Pública, etc.) pone de manifiesto el importante papel desempeñado por el sector empresarial, representando en Euskadi más del 75\% y siendo en la región el agente más relevante. La intensidad de I+D empresarial es pues muy significativa, como se indicó arriba, estando considerada la más importante de España, que de media ejecuta algo más del 50\%, e incluso es superior a la de países europeos líderes en la realización de actividades de I+D (OCDE, 2011; Gálvez, González, 2012; Jordá et al, 2014). Le sigue a distancia la Enseñanza Superior $(17,21 \%$ frente al $27,74 \%$ del conjunto nacional), evidenciando la apuntada debi- 


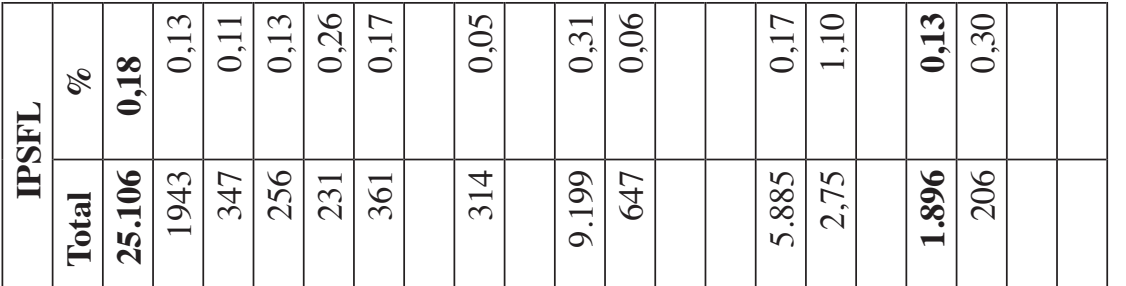

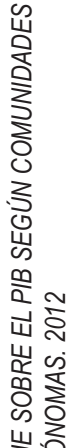

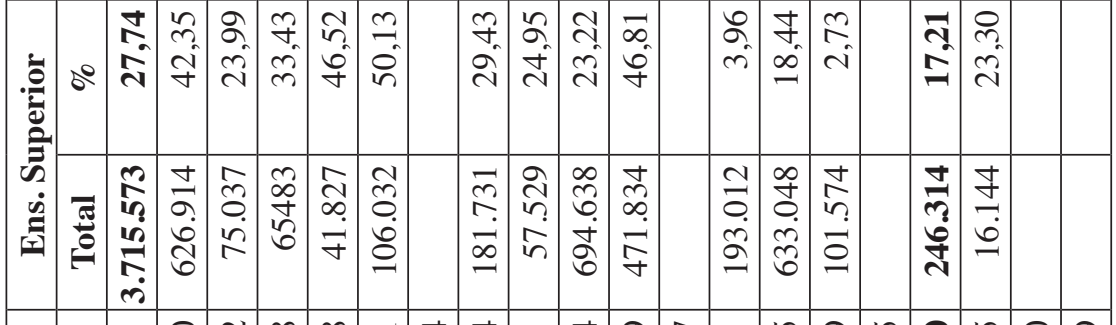

অ

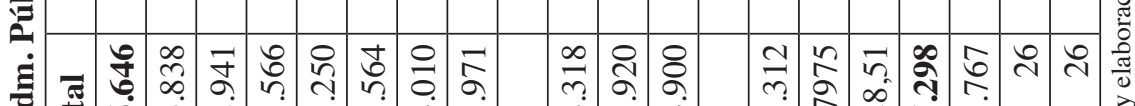

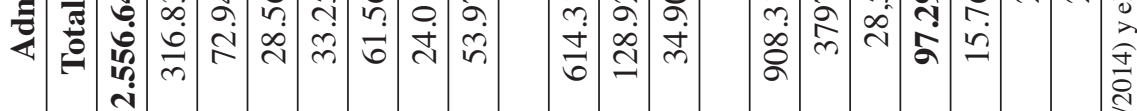

$\pi$ 更

$\frac{\pi}{\circ} \frac{1}{5}$

嵌岕

这岁

c)

岩>

点

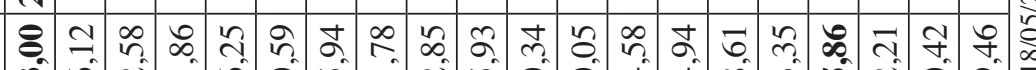

\& ही

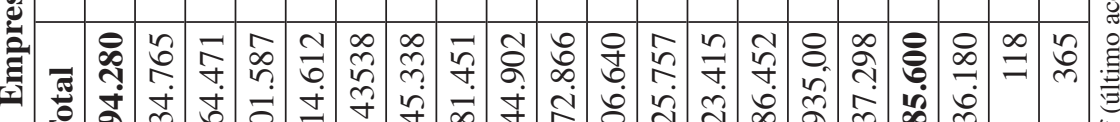

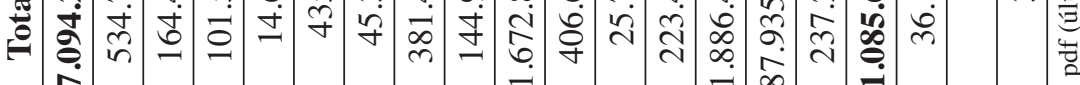

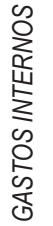

光

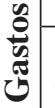

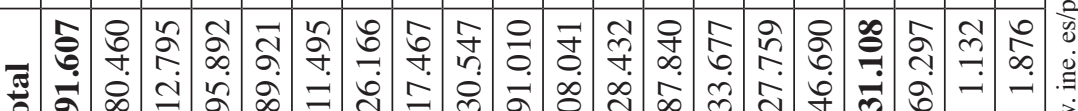

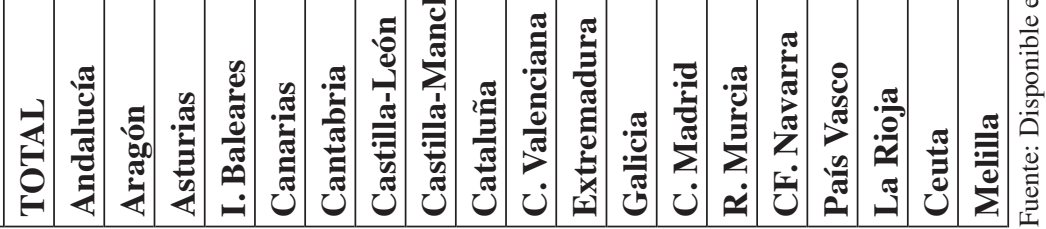


lidad de este agente comparativamente y que en Baleares, Canarias, Comunidad Valenciana y Andalucía, por el contrario, llega a superar el 40\%. En el promedio nacional, la importancia de la Administración Pública también es claramente superior, siendo la responsable de ejecutar casi el $20 \%$ del gasto, despuntando nuevamente los archipiélagos así como Extremadura y Madrid, con ratios superiores, frente al tímido 6,8\% que el sector público representa en Euskadi. En idéntico sentido, los fondos procedentes de otras instituciones (IPSFL - grandes empresas, entidades sin ánimo de lucro, etc.- $)$, son superiores en el conjunto nacional $(0,18 \%$ de media), en un contexto generalizado de escasa relevancia y contando en el País Vasco con una ratio del 0,13\%, frente a la importancia relativa que adquieren en Cataluña o Baleares.

En cuanto a los recursos humanos, y según la misma fuente, la proporción de personal total (E.P.D) en relación con el total de población ocupada de cada región es también superior en Euskadi, ocupando el primer puesto en el ranking autonómico con un 21,63\% de su población ocupada (19.000 efectivos), frente al promedio nacional donde el personal implicado en actividades de I+D representa casi la mitad (12,32\% de la población ocupada total). Navarra, Madrid y Cataluña también destacan a este respecto. El reparto del personal EPD en España desde la perspectiva del total de la población ocupada nacional evidencia con todo que las mayores concentraciones se dan en Madrid y Cataluña llegando entre ambas a absorber casi la mitad del personal dedicado a I+D. Lo mismo sucede si analizamos el porcentaje de investigadores (E.D.P) con un 13,73\% sobre la población ocupada en Euskadi en 2012 (12.000 efectivos frente al 7,48\% que representa en España) y ocupando así mismo el primer puesto, seguido de Navarra, Madrid y Cataluña. Nuevamente estas dos últimas comunidades son las que concentran, en el contexto del conjunto nacional, el mayor volumen de investigadores. En definitiva, los logros en materia de gasto y recursos humanos en I+D son altamente satisfactorios en Euskadi situándose a la cabeza del país.

\section{IV.1.1. Resultados a nivel europeo}

Finalmente y como se comentó, la comparación del esfuerzo inversor a escala de los países de la Unión Europea (Gasto en I+D sobre el \% PIB) refleja que, según los últimos datos disponibles de Eurostat y Eustat, el País Vasco, no sólo está por encima de la media nacional $(1,30 \%)$ sino de la media de la UE-27 que es del 2, 08\% PIB respectivamente en 2012. La CAPV se sitúa así en el décimo puesto de la UE-27 puesto estando delante países como Finlandia (3,55\%), Suecia $(3,41 \%)$, Dinamarca $(2,98 \%)$ Alemania $(2,98 \%)$, etc. en tanto que el valor promedio sitúa a España en un discreto puesto décimo sexto, a la par que Hungría $(1,30 \%)$ y por debajo de países como Luxemburgo $(1,46 \%)$, Portugal $(1,50 \%)$, Irlanda $(1,72 \%)$, etc. Desde una perspectiva evolutiva, Euskadi sigue una dinámica similar a la media de la Unión europea con aumentos anuales todos los años, a diferencia de España donde existen claros altibajos como se apuntó anteriormente, evidenciando una posición bastante rezagada (EUROSTAT (19-05-2014) y EUSTAT (Estadística sobre actividades de investigación científica y desarrollo tecnológico) ${ }^{6}$.

6 [Enlínea] URL:<http://www. eustat. es/elementos/ele0003200/ti_Gasto_en_ID_PIB_por_pais_19972012/tbl0003292_c. html (Consultado el 10/04/2014). 


\section{IV.1.2. Resultados en la Comunidad Autónoma del País Vaco}

Con todo, el importante esfuerzo inversor en I+D en la CAPV no es homogéneo tampoco desde el punto de vista espacial entre los tres Territorios Históricos. Obviamente, tampoco a escala comarcal y local los datos son iguales.

Tabla 3

GASTO EN I+D Y PORCENTAJE SOBRE EL PIB. TOTAL CAPV Y TERRITORIOS HISTÓRICOS

\begin{tabular}{|l|c|c|c|}
\hline & $\mathbf{2 0 1 2}$ & $\mathbf{2 0 1 2}$ & \% sobre PIB (2012) \\
\hline Gastos en I+D & total & \% total & \% total \\
\hline Total (miles de $€$ ) & 1.372 .901 & 100,00 & 2,12 \\
\hline Vizcaya & 628.890 & 45,81 & 1,90 \\
\hline Guipuzcoa & 570.397 & 41,55 & 2,71 \\
\hline Álava & 173.615 & 12,65 & 1,66 \\
\hline
\end{tabular}

Fuente: EUSTAT, Estadística sobre actividades de innovación desarrollo tecnológico, 2014.

Así, la valoración por Territorios Históricos evidencia diferencias notorias (Tabla 3). Vizcaya es la que concentra el mayor volumen de inversión en I+D con el 45,81\% del total (casi 630 millones de $€$ ), seguida a corta distancia por Guipúzcoa con el 41,55\% (más de 570 millones de $€$ ) y ya, bastante más rezagada Álava con el 12,65\% (173 millones de $€$ ). No obstante, el porcentaje del gasto en I+D sobre el PIB, y frente al 2,12\% de media regional según Eustat, enfatiza el esfuerzo realizado en Guipúzcoa, con un 2,71\%, seguida de Vizcaya $(1,90 \%)$ y finalmente Álava $(1,66 \%)$. En ello tiene que ver la perspectiva comarcal, donde la valoración del gasto interno en I+D coloca en primera posición a cuatro comarcas, todas ellas guipuzcoanas: Bajo Deva $(4,88 \%)$, Alto Deva $(4,60 \%)$, Goyerri $(3,20 \%)$ y Donostialdea $(2,67 \%)$. Por otro lado, y de los 1.372 millones $€$ invertidos en actividades de investigación científica y desarrollo tecnológico, cuatro comarcas superaron los 100 millones $€$ de gasto: Gran Bilbao (514 millones, el 37,46\%), San Sebastián (260 millones, el 18,95\%), Llanada Alavesa (154 millones $(11,22 \%)$ y Alto Deva (106, 1 millones $(7,73 \%)$, territorios que sumaron el 75,36\% del gasto regional en I+D (EUSTAT. Estadística sobre actividades de investigación científica y desarrollo tecnológico $I+D)$. En cuanto al personal EDP dedicado a la I+D destacan en el mismo orden Gran Bilbao con el 38\%, seguido de la comarca de San Sebastián (Donostialdea) con el 20,5\% y LLanada Alavesa con el 11,8\%, lo que evidencia una especial concentración de la plantilla implicada en I+D en aquellos espacios comarcales que coinciden con la ubicación y localización de las capitales respectivas de los Territorios Históricos, dominando Bilbao, seguida de Donosti y finalmente Vitoria ${ }^{7}$. Mención aparte destacan las comarcas guipuzcoanas del Alto y Bajo Deva, donde tiene su sede y buena parte de sus instalaciones motrices y de fabricación el Grupo Mondragón Corporación Cooperativa con sede en Mondragón, explicando el elevado esfuerzo en la materia realizado en

7 [Enlínea] URL:http://www. eustat. es/elementos/ele0004900/ti_Gasto_interno__PIB_total_y_personal_EDP_dediacado_a_ID_en_la_CA_de_Euskadi_por_comarcas_2012/tb10004990_c. html (Consultado el $10 / 04 / 2014)$ 
esta comarca como se ha indicado. El análisis de los sectores que realizan las inversiones en I+D pone de manifiesto que, dentro del País Vasco Guipúzcoa destaca por la fortaleza de un diversificado sector empresarial, debido a las características de su tejido productivo y por la mayor presencia de los centros tecnológicos. Vizcaya es el territorio con mayor peso del sector Universidad mientras que en Álava lo es la Administración Pública. En el caso de Vizcaya, la mayor presencia de facultades, en especial de ciencias e ingenierías, explica ese comportamiento. En Álava existe una mayor concentración de organismos de investigación dependientes de la Administración (Gálvez y González, 2012).

\section{IV.2. Visión de la I+D en el País Vasco por agentes del sistema de innovación}

\section{IV.2.1. La innovación en las empresas}

Como ha quedado patente en el apartado anterior, las actividades de I+D son realizadas principalmente por las empresas, especialmente en Euskadi, donde ejecutan casi la mayor parte del gasto en innovación. De acuerdo con la Encuesta de Innovación del Eustat, que recoge el porcentaje de establecimientos de 10 y más empleados que realizan innovación tecnológica en el País Vasco (Tabla 4), de una media próxima al 31,3\% en el período 20102012, sobresale la provincia de Guipúzcoa, con un 34\%, mostrando Alto Deba (41\%), Goyerri (37\%) y la comarca de San Sebastián (35\%), un especial dinamismo en este aspecto. Las comarcas con ratios por encima de la media son también Cantábrica Alavesa $(34,8 \%)$ y Resto de Álava en Álava; Duranguesado, Guernica-Bermeo y Plenzia-Munguia en Vizcaya; y todas las de Guipúzcoa con excepción de Bajo Bidasoa. Con todo, las series históricas evidencian una ligera disminución global del 1,4\% entre 2004 y 2012, mucho más visible en Álava $(-3,8 \%)$ que en Vizcaya y Guipúzcoa $(-0,9 \%$ y $-1,3 \%$ respectivamente) reflejando la mella de la crisis económica (EUSTAT. Encuesta de Innovación (EIT y elaboración propia).

Interesa especialmente la Tabla 5 al mostrar los resultados que ha tenido la innovación en los establecimientos innovadores vascos, medidos éstos según rama de actividad y estrato de empleo durante el periodo 2011-2013. Así, y del total de firmas vascas innovadoras, más del $80 \%$ reconoce haber experimentado algún tipo de efecto derivado de la introducción de innovaciones, vinculándose la mayor parte de los cambios con logros positivos sobre los procesos productivos $(69,6 \%$-mayor flexibilidad y/o capacidad en la producción o prestación de servicios, menores costes laborales por unidad producida, etc.) y los productos $(66,6 \%$-gama más amplia de bienes y servicios, sustitución de productos o procesos anticuados, mayor cuota de mercado, etc.). En cuanto a los efectos sobre el empleo, apuntado por casi el $45 \%$ de las firmas, más de un $30 \%$ de las que han innovado lo ha mantenido, casi un $12 \%$ ha aumentado el empleo cualificado y en torno a un $8 \%$ han incrementado el empleo total, proporción idéntica a las que afirman haberlo reducido. En cuanto a otros efectos, destaca el cumplimiento de los requisitos normativos en el 23,5\% del total y el menor impacto medioambiental y mejora de la salud y seguridad de sus trabajadores. Por ramas de actividad, los establecimientos industriales junto con los vinculados al sector servicios registran efectos derivados de la innovación en un $88,1 \%$ y $83,4 \%$ respectivamente, seguidos a gran distancia de la construcción, donde la proporción de establecimientos es menor si bien supera la mitad de éstos (56,5\%). En torno a tres cuartas 
Tabla 4

PORCENTAJE DE ESTABLECIMIENTOS DE 10 Y MÁS EMPLEADOS QUE REALIZAN INNOVACIÓN TECNOLÓGICA EN LA CAPV. TOTAL, PROVINCIAL Y COMARCAL

\begin{tabular}{|c|c|c|c|c|c|c|c|}
\hline & 2004-2006 & 2005-2007 & 2006-2008 & 2007-2009 & 2008-2010 & 2009-2011 & 2010-2012 \\
\hline & Total & Total & Total & Total & Total & Total & Total \\
\hline C.A. de Euskadi & $32,7 \%$ & $32,0 \%$ & $31,5 \%$ & $29,8 \%$ & $30,1 \%$ & $30,7 \%$ & $31,3 \%$ \\
\hline Vitoria & $33,2 \%$ & $28,9 \%$ & $28,1 \%$ & $29,1 \%$ & $30,7 \%$ & $31,6 \%$ & $29,8 \%$ \\
\hline Bilbao & $29,1 \%$ & $29,0 \%$ & $29,5 \%$ & $27,6 \%$ & $24,6 \%$ & $27,7 \%$ & $29,0 \%$ \\
\hline Donostia & $39,3 \%$ & $35,8 \%$ & $33,4 \%$ & $31,5 \%$ & $34,5 \%$ & $34,5 \%$ & $35,0 \%$ \\
\hline Álava & $34,3 \%$ & $30,1 \%$ & $29,9 \%$ & $30,4 \%$ & $30,1 \%$ & $33,2 \%$ & $30,5 \%$ \\
\hline Llanada Alavesa & $33,0 \%$ & $29,1 \%$ & $28,2 \%$ & $29,1 \%$ & $30,8 \%$ & $31,6 \%$ & $30,1 \%$ \\
\hline Rioja Alavesa & $30,6 \%$ & $29,5 \%$ & $38,2 \%$ & $33,3 \%$ & $35,9 \%$ & $30,8 \%$ & $25,1 \%$ \\
\hline Cantábrica Alavesa & $47,5 \%$ & $40,1 \%$ & $39,8 \%$ & $37,7 \%$ & $37,0 \%$ & $39,7 \%$ & $34,8 \%$ \\
\hline Resto de Álava & $34,4 \%$ & $30,2 \%$ & $31,4 \%$ & $33,7 \%$ & $40,6 \%$ & $43,7 \%$ & $32,8 \%$ \\
\hline Vizcaya & $30,5 \%$ & $29,9 \%$ & $29,4 \%$ & $27,9 \%$ & $25,6 \%$ & $28,2 \%$ & $29,6 \%$ \\
\hline Arratia-Nervión & $35,5 \%$ & $33,1 \%$ & $31,6 \%$ & $31,3 \%$ & $31,8 \%$ & $27,4 \%$ & $28,4 \%$ \\
\hline Gran Bilbao & $29,7 \%$ & $29,0 \%$ & $28,6 \%$ & $26,9 \%$ & $24,6 \%$ & $27,6 \%$ & $28,9 \%$ \\
\hline Duranguesado & $33,1 \%$ & $33,1 \%$ & $32,9 \%$ & $31,4 \%$ & $29,2 \%$ & $30,8 \%$ & $34,0 \%$ \\
\hline Encartaciones & $29,0 \%$ & $30,4 \%$ & $27,8 \%$ & $25,9 \%$ & $22,7 \%$ & $27,2 \%$ & $29,2 \%$ \\
\hline Gernika-Bermeo & $34,4 \%$ & $35,8 \%$ & $35,3 \%$ & $35,2 \%$ & $32,0 \%$ & $31,4 \%$ & $31,8 \%$ \\
\hline Markina-Ondarroa & $33,0 \%$ & $31,5 \%$ & $32,1 \%$ & $30,5 \%$ & $24,7 \%$ & $33,8 \%$ & $29,9 \%$ \\
\hline Plentzia-Mungia & $34,4 \%$ & $31,6 \%$ & $30,9 \%$ & $34,3 \%$ & $33,6 \%$ & $32,6 \%$ & $33,0 \%$ \\
\hline Guipuzcoa & $35,3 \%$ & $36,3 \%$ & $35,4 \%$ & $32,2 \%$ & $\mathbf{3 5 , 8 \%}$ & $33,2 \%$ & $34,0 \%$ \\
\hline Bajo Bidasoa & $29,6 \%$ & $38,2 \%$ & $29,7 \%$ & $25,1 \%$ & $27,4 \%$ & $26,1 \%$ & $28,0 \%$ \\
\hline Bajo Deba & $34,9 \%$ & $39,2 \%$ & $34,7 \%$ & $33,8 \%$ & $39,1 \%$ & $32,6 \%$ & $31,9 \%$ \\
\hline Alto Deba & $37,8 \%$ & $40,7 \%$ & $42,7 \%$ & $36,9 \%$ & $39,2 \%$ & $38,1 \%$ & $41,0 \%$ \\
\hline Donostialdea & $35,7 \%$ & $34,4 \%$ & $34,6 \%$ & $31,7 \%$ & $35,0 \%$ & $33,5 \%$ & $33,8 \%$ \\
\hline Goierri & $36,8 \%$ & $39,2 \%$ & $36,9 \%$ & $34,6 \%$ & $39,8 \%$ & $35,1 \%$ & $37,6 \%$ \\
\hline Tolosa & $34,3 \%$ & $31,8 \%$ & $34,7 \%$ & $33,8 \%$ & $37,3 \%$ & $31,9 \%$ & $34,0 \%$ \\
\hline Urola Costa & $35,8 \%$ & $37,8 \%$ & $38,0 \%$ & $33,1 \%$ & $39,2 \%$ & $34,0 \%$ & $33,6 \%$ \\
\hline
\end{tabular}

EUSTAT. Encuesta de Innovación (EIT) y elaboración propia.

partes de las firmas industriales y de servicios han mejorado aspectos relacionados con los productos. Alrededor del $70 \%$ de las empresas manufactureras y de servicios muestran haber obtenido efectos positivos vinculados con la innovación en procesos, siendo nuevamente menor el reflejo de la innovación en el sector de la construcción. En cuanto a los efectos sobre el empleo, los impactos más positivos se han producido en el sector industrial donde un 35,1\% ha mantenido el empleo, un 23,5\% ha aumentado la plantilla cualificada y un $18,6 \%$ la plantilla total. En la construcción destaca el mantenimiento del empleo $(53,6 \%)$ y en los servicios $(26,1 \%)$. Respecto a los establecimientos innovadores de 10 o más trabajadores, se observa cómo, en líneas generales, las tendencias expresadas arriba para el conjunto se asemejan, con una propensión a agravarse en el sentido de aumentar el porcentaje de firmas que han logrado efectos positivos asociados a la introducción de innovaciones (90,6\% del total), destacando los relacionados con las mejoras en los productos $(85 \%)$ y en los procesos $(70 \%)$. En cuanto al empleo, un $40,6 \%$ lo ha mantenido, un $25,5 \%$ ha aumentado la plantilla cualificada y casi un $18 \%$ ha aumentado el empleo total. Por ramas de actividad, industria y servicios se han visto más beneficiadas (91\% aprox.), si bien la construcción ha aumentado su proporción relativa $(78,4 \%)$. En firmas industria- 


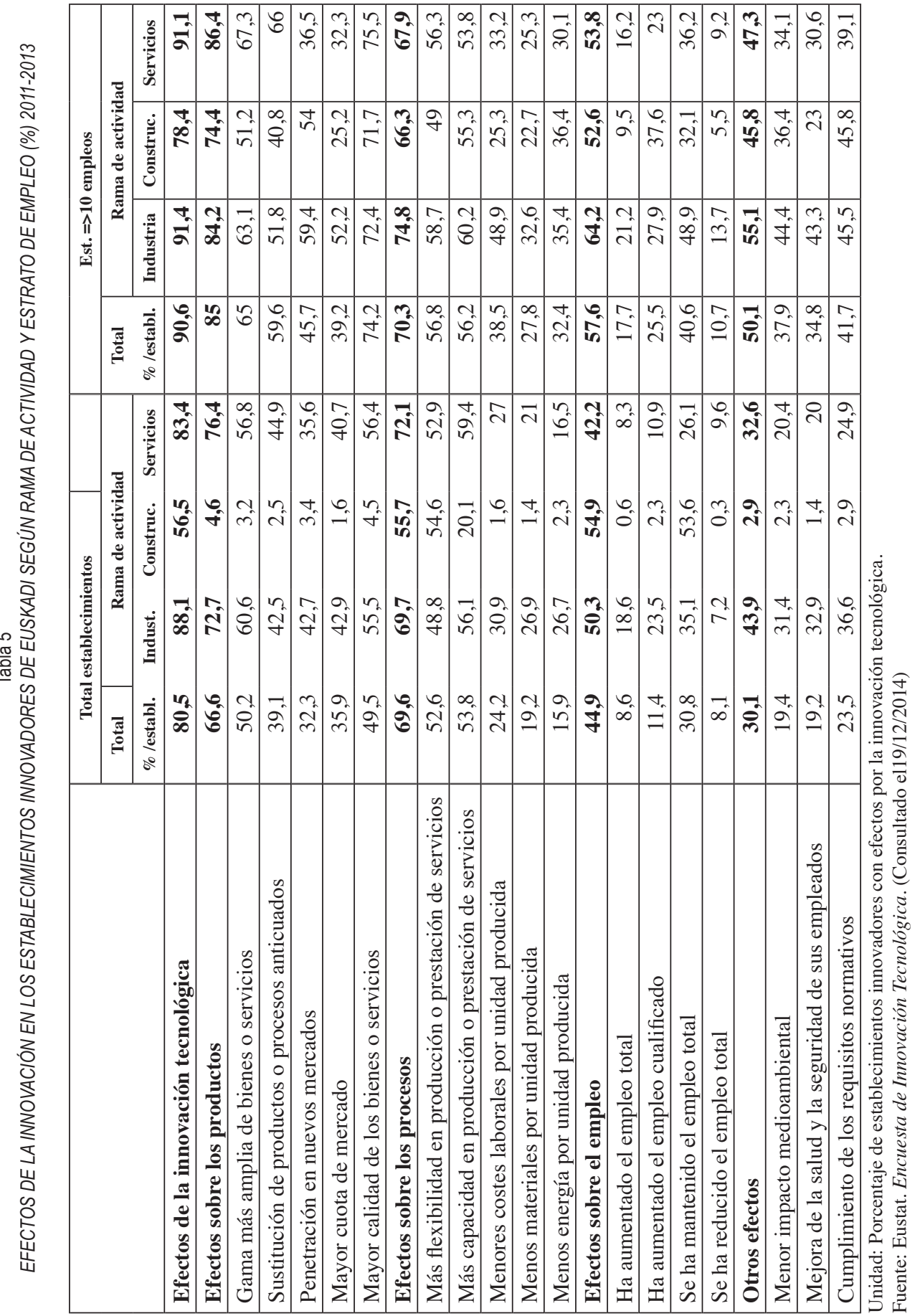


les innovadoras, los efectos positivos sobre los productos, procesos, el empleo, etc. son superiores a los establecimientos de menos de 10 empleados; Tendencia que se reproduce en las de la construcción y los servicios. Por tanto, y pese a la crudeza con que la crisis ha azotado a España en su conjunto, estos datos vienen a confirmar que las empresas innovadoras, esto es, comprometidas con la innovación muestran a las claras que han soportado mejor el impacto recesivo, lo que viene a confirmar el importante papel del sistema de innovación vasco (y fundamentalmente de las empresas en tanto agentes integrantes del mismo) y de la política de apoyo a la I+D en la mejor superación de la crisis y en la ansiada transformación del modelo productivo hacia la economía del conocimiento, como avalan las menores tasa de paro obtenidas en relación con la media estatal y mejoras en múltiples aspectos señalados que apuntalan su competitividad y productividad. Finalmente, y como otro de los indicadores que muestra el mejor desempeño económico del País Vasco durante la crisis, los resultados de la tabla Tabla 6 sobre el balance comercial de los bienes del sector de alta tecnología en Euskadi, resultan claramente positivos para ésta, de modo que el valor de las exportaciones de productos vascos es superior al de las importaciones (casi 21 millones de euros frente a 15.824 respectivamente). Por Territorios Históricos destaca abiertamente Guipúzcoa, la provincia más exportadora. En cuanto al grupo de productos donde la internacionalización es superior destacan la construcción aeronáutica y espacial, la maquinaria de oficina y equipamiento informático, el material electrónico, equipos, y aparatos de radio, TV y comunicaciones; los productos farmacéuticos, instrumentos cien-

Tabla 6

IMPORTACIONES Y EXPORTACIONES DE BIENES DEL SECTOR DE ALTA TECNOLOGÍA EN EUSKADI POR TERRITORIOS HISTÓRICOS Y GRUPOS DE PRODUCTOS (MILES DE EUROS) 2012

\begin{tabular}{|l|r|r|}
\hline & Importaciones & \multicolumn{1}{|c|}{ Exportaciones } \\
\hline Total sectores & $\mathbf{1 5 . 8 2 4 . 6 1 8}$ & $\mathbf{2 0 . 9 7 1 . 1 9 7}$ \\
Sector alta tecnología & 510.152 & 756.678 \\
Territorio histórico & & \\
- Araba / Álava & 120.580 & 65.838 \\
- Bizkaia & 249.469 & 258.368 \\
- Gipuzkoa & 140.103 & 432.472 \\
\hline Grupos de productos & & \\
1. Construcción aeronáutica y espacial & 85.347 & 100.340 \\
2. Maquinaria de oficina y equipo informático & 26.675 & 12.417 \\
3. Material electrónico equipos y aparatos de radio, & & \\
TV y comunicaciones & 162.514 & 90.618 \\
4. Productos farmacéuticos & 5.942 & 5.440 \\
5. Instrumentos científicos & 85.737 & 67.437 \\
6. Maquinaria y material eléctrico & 20.492 & 22.412 \\
7. Productos químicos & 21.912 & 6.143 \\
8. Maquinaria y equipo mecánico & 85.529 & 407.655 \\
9. Armas y municiones & 16.004 & 44.216 \\
\hline
\end{tabular}

Fuente: EUSTAT: Panel de indicadores de Alta Tecnología (Consultado el 19/12/2014). 
tíficos, etc. demostrando así la progresiva diversificación de la estructura productiva hacia sectores de media y alta tecnología en el contexto de la pretendida «Segunda Gran Transformación Económica» del País Vasco hacia la economía del conocimiento y la innovación.

\section{IV.2.2. La I+D en los Centros Tecnológicos}

Junto a las empresas, y en el contexto del SRI vasco, una de sus características es la importante red de centros tecnológicos que posee, fruto de la política tecnológica aplicada por el Gobierno Vasco desde los ochenta tan señalada. Es importante destacar que el Eustat incluye a los centros tecnológicos dentro del sector empresas, de manera que lo expuesto en el epígrafe anterior debe relacionarse con los CT's, pero dado el importante papel que desempeñan en Euskadi como proveedores de servicios de I+D merecen un estudio, aunque breve, diferenciado. Los centros tecnológicos son infraestructuras de carácter privado sin ánimo de lucro que proveen de tecnología de alto valor añadido. Dichos centros se dedican principalmente a la realización de actividades de I+D y, con carácter secundario, prestan servicios de asesoramiento, formación, ensayos industriales y otros de naturaleza tecnológica. Los datos relativos al gasto en I+D de los CT's de Euskadi según el origen de los fondos desde 2010 reflejan un importe de casi 223 millones de $€$ en 2012, lo que supone un aumento frente a 2010 (218 millones) pero una disminución respecto a 2011 (231 mill.), reflejo de los efectos que, sobre las empresas y el presupuesto público está teniendo el periodo recesivo ${ }^{8}$. Los fondos que financian las actividades de investigación de los centros tecnológicos tienen su origen principalmente en el sector empresarial (49,88\%) y en la Administración Pública $(33,00 \%)$, de donde proceden el 83\%. No hay que olvidar la importante relación que mantienen con las empresas y que, asimismo, han estado fuertemente apoyados por la Administración. No obstante, en los últimos años las empresas han aumentado su peso como proveedoras de recursos en menoscabo de los fondos públicos. Los fondos procedentes del extranjero (Otros), fundamentalmente de los programas comunitarios, suponen el 17,12\% de todo el gasto en I+D que realizan en 2012. Conviene destacar que, al igual que ocurre en el sector empresas, la mayoría del gasto en I+D de los centros tecnológicos va dirigido a las disciplinas de Ingeniería y Tecnología, con un $82 \%$ del total, cifra muy alejada de la correspondiente al resto de campos científicos (Gálvez y González, 2012). El personal empleado en los centros tecnológicos ha mostrado una tendencia ascendente, hasta alcanzar los 2.877 empleados en EDP en 2012 lo que supone el 15,25\% del personal que realiza actividades de I+D en la C. A. de Euskadi. En cuanto a la distribución del personal por su ocupación, se observa que más de las tres cuartas partes son investigadores $(76,7 \%)$. De las otras dos categorías, el personal técnico $(15,2 \%)$, aunque ha aumentado, ha perdido importancia relativa a favor principalmente del personal auxiliar $(8,1 \%)$. El 44,14\% del personal de los centros tecnológicos son mujeres, el $64 \%$ investigadoras siendo el resto a partes iguales técnicos y auxiliares. Las mujeres han ido aumentando en número en todos los años analizados ${ }^{9}$.

8 [Enlínea]URL:http://www. eustat. es/elementos/ele0010100/ti_Centros_Tecnologicos_Gasto_interno_en_ ID_en_la_CA_de_Euskadi_por_origen_de_los_fondos_miles_de_euros_2010-2012/tbl0010110_c. html (Consultado el 26/04/2014) y elaboración propia.

9 [Enlínea] URL:http://www. eustat.es/elementos/ele0007500/ti_Centros_Tecnologicos_Personal_EDP_en_ ID_en_la_CA_de_Euskadi_por_ocupacion_y_sexo_2010-2012/tb10007516_c. html (Consultado el 28/04/2014) y elaboración propia. 
Como se apuntó arriba, el Gobierno Vasco ha promovido el agrupamiento de los principales centros tecnológicos en dos redes, Tecnalia e IK-4. La corporación Tecnalia está compuesta de 20 unidades empresariales y una plantilla de 1.415 empleados en 2012 (200 doctores), 21 sedes en todo el mundo, más de 4.000 clientes y unos ingresos totales de 102 millones de $€$, situándose entre las cinco mayores corporaciones europeas de tal naturaleza, 303 patentes en cartera y 15 nuevas empresas de base tecnológica. Con todo, estas cifras se han visto menguadas por la crisis (Gerencia TENALIA). Por su parte, $I K-4$ es una alianza de siete centros:AZTERLAN, CEIT, CIDETEC, GAIKER, IDEKO, IKERLAN, TEKNIKER, LORTEK y VICOMTech. Tiene 1. 400 empleados (23\% doctores), retornos europeos por valor de 78 millones de $€, 145$ patentes en curso y 833 clientes privados y unos ingresos anuales de más de 108 millones de $€$ en 2012 (Fuente: Gerencia).

En una valoración global sobre los centros tecnológicos, éstos han desempeñado un papel fundamental en la transferencia de conocimiento tecnológico y de innovación hacia el sector empresarial, como se deduce al analizar los efectos de la innovación en las empresas en el epígrafe anterior, si bien los resultados de la colaboración con éstas y otros agentes del sistema no siempre han sido suficientemente satisfactorios. Por un lado, existen problemas de comunicación que se manifiestan en la dificultad que tienen las empresas a la hora de definir sus problemas tecnológicos y en la insuficiente articulación de sus demandas tecnológicas. También está la dificultad que tienen los centros tecnológicos para captar las necesidades de las empresas, debido a que éstas sienten recelos a descubrir sus debilidades. Asimismo, una de las principales dificultades es acceder a las empresas. Las empresas vascas, en general pymes poco innovadoras, no tienen suficiente conciencia de la importancia de la I+D y no se involucran en proyectos sin el apoyo de la Administración. La forma ideal de cooperación entre empresas y centros tecnológicos es la formación de grupos y equipos mixtos integrados por personas de la empresa y de los centros tecnológicos, lo que facilita la asimilación de tecnología por la empresa. Sin embargo, en la práctica esta colaboración no ha sido demasiado frecuente (Olazarán y otros, 2007, 2009). Otra cuestión a destacar es la poca conexión entre los centros tecnológicos y la Universidad, consecuencia en parte de la falta de coordinación entre la política tecnológica (que depende del Departamento de Industria) y la política científica (que depende del Departamento de Educación). Desde los centros tecnológicos se reconoce que las relaciones con la Universidad son muy escasas, y aunque por un lado se afirma que el papel de los centros tecnológicos debe ser de intermediación entre la universidad y la empresa, por otro lado se les ve como competidores en la percepción de fondos, por lo que surgen problemas para la cooperación (fuente: ídem). De cara a reforzar la eficiencia del SRI sería importante aumentar esa coordinación (algo común en otros países europeos) teniendo en cuenta que los perfiles de actividad de ambos agentes son bastante complementarios.

\section{IV.2.3. Universidades y Administración Pública}

Las Universidades junto a los Organismos de Investigación dependientes de la Administración Pública forman el núcleo básico del sistema de investigación científica y desarrollo tecnológico del SIR vasco como se ha indicado. En la CAPV existe una gran universidad pública, la Universidad del País Vasco (UPV/EHU), y dos universidades privadas (Deusto y Universidad de Mondragón). Los estudiantes vascos también tienen acceso a la Universidad 
de Educación a Distancia (UNED) con tres centros adscritos, así como a algunas facultades de la Universidad de Navarra situadas en Euskadi (Tecnum). La UPV/EHU es la universidad más grande en cuanto al número de estudiantes (76\% de todo el alumnado), investigadores y unidades de investigación y posee la acreditación de Campus de Excelencia Internacional mediante el proyecto Euskampus, una iniciativa conjunta con la Fundación Donostia Internacional Physics Center (DIPC) y la corporación Tecnalia. El gasto en actividades de $\mathrm{I}+\mathrm{D}$ realizado por las universidades vascas ha seguido una tendencia ascendente a lo largo de la década del 2000 (Instituto Vasco de Competitividad, 2013). No obstante, el sector universitario presenta una debilidad comparativa notable a este respecto si se considera que el nivel de gasto que ejecutan supone tan sólo el $18 \%$ del gasto total de I+D de la CAPV, participación inferior a la media española $(27,74 \%)$ y europea $(24 \%)$ como se comentó; Con todo, esta pérdida de peso relativa se ha visto compensada por la aparición de los $B E R C$, que se contabilizan en este mismo sector en las estadísticas, pero es mayor si tenemos en cuenta que en la categoría de centros de enseñanza superior también está computada igualmente la actividad de Ikerbasque cuyos investigadores trabajan para la Universidad (Fuente; ídem). Las actividades de I+D de las universidades se financian principalmente con fondos públicos $(75,15 \%)$. Se trata de recursos captados a través de convocatorias públicas competitivas de Euskadi, o sea, de la Administración autonómica (Departamento de Educación y Departamento de Industria del Gobierno Vasco, a través de los programas Saiotek y Etortek) y de los Planes Nacionales de I+D+i, así como de los servicios ofrecidos a las Administraciones públicas. El 13,86\% del gasto en investigación se financia con fondos procedentes de las propias universidades, mientras que las empresas, a través de contratos, financian el 6,09\% de las actividades investigadoras de las universidades. Los recursos provenientes del extranjero, básicamente vía participación en proyectos de los Programas Marcos de la UE, apenas suponen el 4,6\% si bien han aumentado en los últimos años (Orkestra, 2013; Gálvez y Gonzalez, 2012). Actualmente, desde 2012 y por efecto de la crisis la financiación pública ha disminuido, lo que supone un reto para las organizaciones vascas que les obliga a explotar otras fuentes de financiación y a realizar ajustes y racionalizaciones. En relación con los recursos humanos las universidades vascas emplean en 2012 a algo más de 4.000 personas dedicadas a actividades de I+D en EDP. El 87,08\% de estos empleados son investigadores, de los cuales son mujeres el 47,62\% (41,47\% del total del personal EDP). No obstante, el grupo de investigadores ha reducido su peso en el total debido al mayor aumento del personal técnico $\mathrm{y}$, principalmente, del personal auxiliar ${ }^{10}$. Con relación al campo de investigación al que van dirigidos los recursos financieros van destinados sobre todo a ingeniería y tecnología (15.100 millones de $€(43,5 \%$ del gasto) y las ciencias sociales y humanidades (11.691 millones (33,69\% del gasto), que es el área que más ha aumentado su peso a años anteriores. Se trata, por otro lado, de un campo científico al que las empresas apenas destinan recursos. Casi un $16 \%$ se destina al campo de las ciencias exactas y naturales ${ }^{11}$.

10 [Enlínea]URL:<http://www. eustat. es/elementos/ele0010900/ti_Personal_EDP_dedicado_a_ID_en_la_ CA_de_Euskadi_por_sector_de_ejecucion_segun_ocupacion_y_sexo_2012/tb10010998_c. html> (Consultado el 28/04/2014) y elaboración propia.

11 [Enlínea]URL:<http://www. eustat. es/elementos/ele0000200/ti_Gasto_interno_en_ID_en_la_CA_de_Euskadi_por_la_disciplina_cientifica_segun_el_origen_de_los_fondos_miles_de_euros_2012/tbl0000235_c. html> (Consultado el 28/04/2013) y elaboración propia. 
En una valoración de conjunto sobre la enseñanza superior en el País Vasco y su contribución a la transformación del modelo productivo, y como señala el Instituto Vasco de Competitividad, la presencia de universidades públicas y privadas es un factor de riqueza, competencia y diferenciación que debería explotarse y potenciarse con más firmeza, pues al igual que la universidad española, la vasca no figuran en las primeras posiciones de los rankings internacionales como el Indice de Shanghai. En 2012 por primera vez la UPV se colocó en el intervalo 301-400 de entre 20.000 instituciones de enseñanza superior evaluadas en el citado ranking (Orkestra, 2013). En lo relativo a la docencia, la universidad vasca presenta una baja ratio alumnos/profesor que, lejos de considerarse un indicador de calidad, es un efecto del exceso de oferta y de la falta de ajuste en la organización a la demanda existente (Pérez y Serrano, 2012). Otro aspecto de suma importancia para la transformación productiva es su capacidad para facilitar procesos de aprendizaje a lo largo de toda la vida partiendo de la asociación positiva entre aprendizaje continuo y output innovador (Lundvall B. A. et al, 2008). En este sentido, y como reconoce el Gobierno Vasco (2012), queda mucho por hacer en las universidades vascas, si bien en las privadas se observan avances más notables en esa dirección. En cuanto a la investigación, la universidad vasca presenta una debilidad relativa, tanto en términos cuantitativos como cualitativos, especialmente la privada en investigación básica (ODCE, 2011). Respecto a la transferencia de conocimiento y tecnología al sistema productivo y territorial, con excepción de la Universidad de Mondragón apenas desarrolla esta faceta. Su papel en el sistema de innovación y en el diseño y dinamización de las estrategias de especialización inteligente ha sido poco relevante. Todo ello es debido a un menor y menos eficiente uso de los recursos financieros ${ }^{12}$. Por otro lado, y como con los CTs, es preciso aumentar la densidad de las relaciones no sólo con éstos, sino con las empresas y el resto de agentes del sistema.

Junto a las empresas y Universidades, y por lo que se refiere al tercer sector ejecutor del gasto en actividades de I+D, esto es, el sector Administración Pública, está constituido por los organismos públicos de investigación (OPI’s) y otras organizaciones dependientes de la Administración. Los principales OPI's de la CAPV son NEIKER -dedicado a la investigación y mejora agraria-, el Centro para el Ahorro y Desarrollo Energético y Minero $(C A D E M), I H O B E$-que se ocupa de la gestión ambiental-, los tres ubicados en Vizcaya; además de los hospitales del Servicio Vasco de Salud repartidos por las tres capitales vascas. En la última década, y como se apuntó en el segundo apartado, se han creado dos tipos de infraestructuras científicas para mejorar el tejido científico e investigador del sistema de innovación vasco: los Centros de Investigación Básica y de Excelencia $(B E R C)$ y los Centros de Investigación Cooperativa $(C I C)$. Los BERC, gestionados por el Dpto. de Educación del Gobierno Vasco, fueron creados para generar una masa crítica de excelencia en la investigación, atraer talento y posicionar a la CAPV en redes globales de investigación, ayudando a romper la endogamia del sistema vasco, a través de sociedades independientes (asociaciones y fundaciones). Por ahora las entidades constituidas como BERC son el Donostia International Physic Center (DIPC), la Unidad de Biofísica de la UPV/EHU, el BERC en Física de Materiales, el BERC en Cambio Climático, el BERC en Matemáticas Aplicadas y el BERC

12 Cabría referirse por ultimo al alto grado de endogamia y falta de internacionalización de las universidades vascas y al marcado carácter local de los investigadores y profesorado docente y del alumnado, exceptuando el perteneciente a los estudios de postgrado y doctorado donde hay una cierta masa crítica de estudiantes latinoamericanos (Gobierno Vasco, 2012; Pérez y Serrano, 2012). 
en Lingüística. Recientemente se han creado otros tres BERC en neurociencia, nuevos materiales y diseño macromolecular representando un total de nueve en la actualidad con una mayor concentración espacial relativa en Vizcaya. En general la colaboración de los BERC con el personal investigador de las universidades ha sido buena, y el personal de los BERC se ha implicado en cursos de posgrado, reforzando la universidad vasca más que sus equivalentes en otros territorios como Cataluña (Orkestra, 2013). Sin embargo, en la mayoría de los casos, las relaciones con otros agentes del sistema de la RVCTI son escasas. Además, aunque han generado spin offs, patentes, etc., en general la transferencia de tecnología ha sido débil. Los CIC, gestionados por el Dpto. de Industria, son centros híbridos creados para desarrollar una investigación en sectores considerados estratégicos para la Comunidad a semejanza de los existentes en EEUU, Canadá, o Suecia, y tratando de acercar la universidad a la industria. Hasta el momento se han establecido siete CIC's: CICbiomaGUNE (Investigación en Biomateriales), CICmarGUNE (Mecanizado de Alto Rendimiento), CICmicroGUNE (MicroNanoTecnologías), CICnanoGUNE(Nanociencias), CICenergiGUNE (Energía) y, finalmente, CICtourGUE (Turismo), en su gran mayoría ubicados en la capital guipuzcoana. Con excepción de CIC Nanogune, su vinculación con la Universidad es escasa. Aunque su misión consista en realizar actividades de investigación orientadas a las necesidades industriales y sociales, lo cual está íntimamente ligado a la transformación productiva, la realidad es bien diferente y la transferencia de tecnología es exigua, excepto en el caso de CICMargune y CICMicrogune. Finalmente, son deficitarios en materia de cooperación. Aunque en sus órganos de gobierno están presentes otros agentes del sistema (universidad y centros tecnológicos fundamentalmente), no hay una efectiva cooperación en investigación haciendo que se pierdan sinergias fundamentales para los procesos de transformación productiva, con diferencias entre centros (ídem:110). Estos organismos dependientes de la Administración Pública tienen en la C. A. de Euskadi poco peso pues apenas ejecutan el 6,31\% del gasto en I+D, si bien es el sector que más ha crecido en los últimos años. Su importancia relativa es menor que la registrada en la UE-27 (13,7\%) y, especialmente, en el conjunto de España (20\%) (Galvez C. y González A, 2012). Por otro lado, Euskadi destaca de nuevo, por ser la comunidad en que este sector tiene menor presencia, situación que contrasta con la que se produce en Madrid, debido principalmente a la presencia del CSIC, que es el mayor organismo público de investigación de España. La financiación de estos OPI's depende de las transferencias de fondos públicos a los que se suman, como en las universidades, los recursos captados externamente a través de convocatorias públicas competitivas, tanto de la CAPV como del Estado, y de los servicios ofrecidos a las Administraciones Públicas. Como consecuencia, son fondos públicos el 88,53\% de los recursos invertidos en actividades investigadoras. De los contratos con el sector privado, que ha aumentado notablemente su aportación, proceden casi el 6,83\%, mientras que ha retrocedido la financiación extranjera. Los recortes de las Administraciones han agravado esta situación, con diferencias entre unos y otros.

\section{CONCLUSIONES}

El País Vasco es una de las regiones que más y mejor ha resistido comparativamente la llegada de la crisis actual. En la explicación de este fenómeno coexisten un buen cúmulo de factores, pero junto a la aplicación de diversas políticas y medidas, y a la existencia de 
determinadas condiciones de partida, se ha destacado el apoyo sostenido y continuado a la innovación durante años por parte de empresas e instituciones como un factor clave, además de constituir un elemento diferenciador en el conjunto, viendo asimismo en la I+D+i una potente estrategia de transformación económica y social capaz de posicionar a Euskadi entre las regiones españolas y europeas más avanzadas. En este marco, el presente texto presenta un propósito de carácter doble: Por un lado, valorar el papel que dichas infraestructuras y agentes integrantes del SRI vasco han desempeñado en la transferencia de conocimiento y en la generación de capacidades en sus empresas para acometer mejor el proceso de transformación productiva apuntado y sortear con éxito los embates de la crisis. Y por otro, relacionado con el anterior, ir un poco más allá y verificar en la medida de lo posible y a partir de la aportación de evidencias, si verdaderamente ese rol y esfuerzo inversor en I+D realizado ha tenido un efecto y unos resultados relevantes en la relativa mejor respuesta a la crisis que ha experimentado la región en el contexto referenciado de impulso a la innovación y transformación de su modelo productivo. Tras analizar en detalle el mejor desempeño económico del País Vasco en el marco estatal (e incluso en parte del ámbito de la UE), y caracterizar el origen, trayectoria y singularidades del SRI vasco en el contexto de las políticas y planes acometidos por las instituciones públicas al respecto, se ha llegado a las siguientes conclusiones: El Sistema de Innovación Vasco está considerado entre los mejores del estado y goza de un gran reconocimiento nacional e internacional. El análisis de los indicadores de innovación, tanto en lo que se refiere al gasto como a los recursos humanos dedicados a actividades de I+D, revela un posicionamiento de Euskadi altamente positivo, presentando un nivel relativo superior al promedio español. La inversión en I+D no ha dejado de crecer en un solo momento, mostrando a las claras la apuesta por un modelo de desarrollo claramente orientado a la innovación en términos de excelencia. Respecto al comportamiento por Territorios Históricos, casi la mitad de los gastos de I+D de la C. A. de Euskadi se concentran en Vizcaya; sin embargo, es en Guipúzcoa donde se realiza el mayor esfuerzo inversor. A su vez, Guipúzcoa destaca por la fortaleza del sector empresarial, mientras que Vizcaya es el territorio en el que tiene mayor peso el sector universitario y en Álava la Administración Pública. La estructura de gasto en I+D por parte de los principales agentes del sistema de innovación evidencia el importante papel desempeñado por el sector empresarial. La intensidad de I+D empresarial está considerada la más importante de España, incluso superior a la de países europeos líderes en actividades de I+D. Y así, el análisis del papel y los efectos del SRI sobre las empresas, revela datos concluyentes y positivos tanto desde la óptica de su mejor conducta respecto a la crisis (empleo, exportación, etc.) como en lo relacionado con la ansiada transformación del modelo económico hacia sectores de mayor contenido tecnológico y valor añadido, resultando la innovación empresarial un pilar fundamental en la salida de la crisis y su menor impacto relativo en la región. Con todo, el análisis del rol de los distintos integrantes del SRI vasco pone al descubierto igualmente diferencias importantes entre sus integrantes, destacando las empresas innovadoras y los centros tecnológicos como los elementos más activos del sistema, si bien el nivel de cooperación entre todos los agentes innovadores en la CAPV no ha alcanzado todavía un umbral de desarrollo adecuado. Entre las principales debilidades o fallos del sistema, las interrelaciones entre las empresas y las infraestructuras tecnológicas (Centros tecnológicos y CIC's) y científicas (Universidad y BERC's) no han llegado a tener el nivel de densidad deseable para generalizar los comporta- 
mientos innovadores al conjunto de las empresas vascas. El aumento de las relaciones es en consecuencia un factor crítico en la mejora del entorno innovador de las empresas y de los propios centros de enseñanza superior. Resulta necesario, por tanto, potenciar más las cooperaciones entre todos los agentes del SRI impulsando actuaciones de mejora a este respecto.

\section{BIBLIOGRAFÍA Y FUENTES}

ALBERTOS, J. M. y SÁNCHEZ J. L. (Coords.): Geografía de la crisis económica en España. Universitat de Valencia. 2014, Valencia.

ANDERSON, M. y KARLSSON , C. (2004): «Regional Innovation Systems in Small \& Medium-Sized Regions», A Critical Review \& Assessment. CESIS Electronic Working Paper Series, 10.

ARANGUREN, MJ.; MAGRO, E.; NAVARRO, M. y VALDALISO, J. M. (2012): Estrategias para la construcción de ventajas competitivas regionales. El caso del País Vasco. Marcial Pons, Madrid.

ARANGUREN, M. J.; NAVARRO, M. y PEÑA, I. (2013): ¿Una crisis y una salida diferentes? El caso de la Comunidad Autónoma del País Vasco, en Papeles de Economía Española, $\mathrm{n}^{\circ} 138,160-177$.

BANDRÉS, E. y GADEA, M. D. (2013): «Crisis económica y ciclos regionales en España», en Papeles de Economía Española, $\mathrm{n}^{\circ}$ 138, 2-29, Funcas-Madrid.

BOSCHMA, R. (2005): «Role of proximity in interaction and performance: Conceptual and empirical challenges», en Regional Studies, vol. 39 (1), 41-45.

DOLOREUX, D. (2002): «What we should know about regional systems of innovation» Technology in Society, 24, 243-263.

GÁLVEZ, C. y GONZÁLEZ, A. (2012): «Ciencia y Tecnología», en Informe socio-económico de Euskadi 2012, n $^{\circ}$ 14, Instituto Vasco de Estadística.

EUSTAT (2014): Estadística sobre actividades de investigación científica y desarrollo tecnológicoen:<http://www. eustat. es/estadisticas/tema_179/opt_0/ti_Estadistica_sobre_ actividades_de_investigacion_cientifica_y_desarrollo_tecnologico/temas.html>

FUNCAS (2013): La economía de las regiones españolas en la crisis, en «Papeles de Economía Española», núm. 138, Funcas-Madrid, 181 pp.

FORAY, D. (2004): The economics of knowledge. The MIT Press, Boston.

GIL E. y SANCHEZ, J. L. (2013): San Sebastián: de la recualificación urbana a los espacios de la innovación. V Jornadas de Campo de Geografía Económica. AGE. Inédito.

GIL, E. (2014): «El País Vasco y su capacidad diferencial de respuesta a la crisis» en Geografía de la crisis económica en España. Universitat de Valencia, pp. XXX.

GILLY, A. y TORRES, A. (Dirs.) (2000): Dynamiques de proximité. L'Harmattan, París.

Gobierno Vasco (2011): Plan de Ciencia, Tecnología e Innovación 2015. Gobierno Vasco. Presidencia. Vitoria-Gasteiz. 2011. Documento técnico.

GOBIERNO VASCO (2012): Plan Universitario 2011-2014. Vitoria. Servicio de Publicaciones.

INE(2012):EstadísticasobreactividadesdeI+D. 2012en:http://www. ine. es/jaxi/menu. do?ty pe $=$ pcaxis $\&$ file $=$ pcaxis $\&$ path $=\% 2 \mathrm{Ft} 14 \% 2 \mathrm{Fp} 057 \% 2 \mathrm{~F} \% 2 \mathrm{Fa} 2012$.

INNOBASQUE (2011). Estudios de la OCDE sobre innovación regional. País Vasco. España. Agencia Vasca de Innovación. 
INEBASE (2014): Contabilidad Regional de España. Base 2008. Principales resultados. [En línea]:http://www. ine. es/jaxi/menu. do?type=pcaxis \&path=\%2Ft35/ p010\&file $=$ inebase $\& \mathrm{~L}=0$

INSTITUTO VASCO DE COMPETITIVIDAD (2013): Informe de competitividad del País Vasco: Transformación productiva para el mañana. Publicaciones Universidad de Deusto. Bilbao.

JORDÁ, R. (2014): «Caracterización del sistema nacional de innovación español (SIN) y de los SRIS durante la crisis», en Albertos, J. M. y Sánchez J. L. (Coords.): Geografía de la crisis económica en España. Universitat de Valencia, Valencia, 263-303.

KOSCHATZKY, K. (1997): «Technology Based Firms in the innovation Process», en Management, Financingand the Regional Networks. Heildelberg.

LÓPEZ-RODRÍGUEZ, J.; FAÍ̃NA, A. y MANSO, G. (2010): «Sistemas de innovación regionales: el caso del País Vasco», Revista Galega de Economía, núm. Extraordinario 19, Universidad de La Coruña.

LUNDVALL, B. (1992): National Systems of Innovation: Towards a Theory of Innovation and Interactive Learning. London: Pinte

LUNDVALL B. A, RASMUSSEN, P. y LORENZ, E. (2012): «Education in the learning economy: a European perspective», en Policy Futures in Education, 6(6), 681-700.

MÉNDEZ, R., SANCHEZ, S., ABAD, L. y GARCÍA, I. (2009): «Sistema urbano y sociedad del conocimiento: hacia una tipología de ciudades españolas», Investigaciones Regionales, $\mathrm{n}^{\circ} 16,117-142$.

MÉNDEZ, R. (2013): «Economía del conocimiento y nuevos contrastes en España: Una perspectiva multiescalar», Boletín de la Asociación de Geógrafos Españoles, $\mathrm{n}^{\circ}$ 63, 7-32.

MORGAN, K (2013): Basque Country RIS3 expert assessment. An expert assessment on behalf of DG Regional and Urban Policy Contract. No. CCI2012CE160ATO58 (marzo).

PORTER, M. (1990): The Competitive Advantage of Nations. New York: Macmillan.

PORTER, M. (1999): Ser competitivo. Bilbao, España: Ediciones Deusto.

NAVARRO, M. (2009): «Los sistemas regionales de innovación: una revisión crítica», en Ekonomiaz Revista vasca de Economía, n ${ }^{\circ}$ 70, 2009, 25-59.

NAVARRO, M. (Dir.) (2013): Las infraestructuras de conocimiento: El caso vasco desde una perspectiva internacional, en Cuadernos Orkestra, 2013/3. Fundación Deusto.

NELSON, R. (1993): National Innovation Systems. A comparative Analysis. Oxford University Press.

OECD (2009): Reviews of Tertiary Education. Spain. Paris. OECD.

OECD (2011): Reviews of Regional Innovation: Basque Country. Sapin. OECD Publisching.

ORKESTRA (2012): Estrategias para la construcción de ventajas competitivas regionales: El caso del País Vasco. Ed. Marcial Pons.

ORKESTRA (2013): Informe de Competitividad del País Vasco 2013. Transformación productiva para el mañana. Publicaciones Universidad de Deusto. Bilbao. 2013.

PERROUX, F (1995): «Note sur la notion de pôle de croissance», en Économie Appliquée, $\mathrm{n}^{\mathrm{o}} 1(2), 83-95$.

PEREZ F. Y SERRANO I. (Dirs.) (2012): Universidad, universitarios y productividad en España. Bilbao. Fundación BBVA.

UNESCO (2005): Towards knowledge societies. Unesco Publishing. Paris. 